\title{
Extending Families of Curves over Log Regular Schemes
}

\author{
by Shinichi Mochizuki
}

\begin{abstract}
In this paper, we generalize to the "log regular case" a result of de Jong and Oort which states that any morphism (satisfying certain conditions) from the complement of a divisor with normal crossings in a regular scheme to a moduli stack of stable curves extends over the entire regular scheme. The proof uses the theory of "regular log schemes " - i.e., schemes with singularities like those of toric varieties - due to K. Kato ([9]). We then use this extension theorem to prove that (under certain natural conditions) any scheme which is a successive fibration of smooth hyperbolic curves may be compactified to a successive fibration of stable curves.
\end{abstract}

1991 Mathematics Subject Classification: Primary subject: 14H10; Secondary Subject: $14 \mathrm{E} 15$.

\section{$\S 0$. Introduction}

In [9], K. Kato introduced the notion of a pair $\left(X, D_{X}\right)$ with toric singularities. Roughly speaking, such a pair consists of a normal scheme $X$ and a divisor $D_{X} \subseteq X$ in that scheme whose singularities are modeled on the same sort of combinatorial data as that classically used to describe toric varieties (as in [17]). Unlike the case of toric varieties, however, where there is a base field, the notion of toric singularities as in [9] is absolute, and hence does not require one to work over any specific base. Moreover, Kato associates to such a pair $\left(X, D_{X}\right)$ a log scheme $\left(X, M_{X}\right)$ (as in [8]). Pairs $\left(X, D_{X}\right)$ with toric singularities correspond to $\log$ schemes $\left(X, M_{X}\right)$ which are log regular. Thus, in particular, toric varieties over a field define (log) regular log schemes - in fact, the theory of regular log schemes which are varieties over a field is essentially equivalent to the theory of varieties with toroidal singularities (i.e., varieties that are étale locally isomorphic to toric varieties) over that field. Alternatively, one may think of $\log \operatorname{regular}\left(X, M_{X}\right)$ as a sort of natural "logarithmic generalization" of the classical notion of a pair $\left(X, D_{X}\right)$ where $X$ is regular and $D_{X}$ is a divisor with normal crossings.

In [7], it is shown that:

If $X$ is regular, and $D_{X}$ is a divisor with normal crossings in $X$, then any morphism from $U_{X} \stackrel{\text { def }}{=} X-D_{X}$ to a moduli stack $\overline{\mathcal{M}}_{g, r}$ of pointed 
stable curves (cf. [3], [10]) that extends to the generic points of $D_{X}$ and maps $U_{X}$ into the open substack $\mathcal{M}_{g, r} \subseteq \overline{\mathcal{M}}_{g, r}$ of smooth curves necessarily extends to a morphism $X \rightarrow \overline{\mathcal{M}}_{g, r}$.

In the present paper, we generalize this result of de Jong-Oort to the case of pair $\left(X, U_{X}\right)$ with arbitrary toric singularities:

Theorem A. (Extension Theorem) Let $(X, M)$ be a regular log scheme. Let $U_{X} \subseteq X$ be the interior of $X$, i.e., the open subscheme where the log structure $M$ is trivial. Write $\overline{\mathcal{M}} \stackrel{\text { def }}{=} \overline{\mathcal{M}}_{g, r}, \mathcal{M} \stackrel{\text { def }}{=} \mathcal{M}_{g, r}$. Let $\overline{\mathcal{M}}^{\mathrm{c}}$ be the coarse moduli space associated to $\overline{\mathcal{M}}_{g, r}$. Suppose that we are given a morphism

$$
h_{U_{X}}: U_{X} \rightarrow \overline{\mathcal{M}}
$$

which satisfies $h_{U_{X}}\left(U_{X}\right) \subseteq \mathcal{M}$. Let us denote by $h_{U_{X}}^{\mathrm{c}}: U_{X} \rightarrow \overline{\mathcal{M}}^{\mathrm{c}}$ the composite of $h_{U_{X}}$ with $\overline{\mathcal{M}} \rightarrow \overline{\mathcal{M}}^{\mathrm{c}}$. Then:

(1) If $h_{U_{X}}$ extends over the generic points of $D_{X}$, then: (i) $h_{U_{X}}^{\mathrm{c}}$ extends to $X ;$ (ii) there exists a surjective, quasi-finite, log étale morphism $(Y, N) \rightarrow(X, M)$ (where $(Y, N)$ is log regular) such that $h_{U_{X}}$ extends over $Y$.

(2) If $h_{U_{X}}$ extends over all points of $X$ of height $\leq 2$, and $X$ is a local complete intersection, then $h_{U_{X}}$ extends to $X$.

(3) If for some $l \geq 3$ which is invertible on $X$, $h_{U_{X}}$ admits a level $l$ structure (cf. Definition 5.6), then $h_{U_{X}}$ extends to $X$.

Theorem A is given as Theorem 6.1 in the text. It generalizes Theorem 5.1 of [7], but we use this result of [7] in the proof of Theorem A (so we do not obtain a new proof of the main result of [7]). Indeed, the proof of Theorem A runs as follows: If $(X, M)$ is $\log$ regular, then (étale locally on $X)$ one may blow-up $(X, M)$ to obtain a log regular $(Y, N)$ such that $Y$ is regular and the divisor $D_{Y}$ where the $\log$ structure $N$ is nontrivial is a divisor with normal crossings. Then one would like to apply the main result of [7] to $(Y, N)$. Unfortunately, this cannot be done immediately because when one restricts the original morphism $h_{U_{X}}: U_{X} \rightarrow \overline{\mathcal{M}}$ to $U_{Y}$, the resulting morphism $h_{U_{Y}}: U_{Y} \rightarrow \overline{\mathcal{M}}$ no longer satisfies the same hypotheses as $h_{U_{X}}$ at the generic points of the divisor $D_{Y}$.

In order to get around this technical difficulty, it is necessary to make use of the log purity theorem: 
Theorem B. (Log Purity Theorem) Let $(X, M)$ be a regular log scheme. Let $U_{Y} \rightarrow U_{X}$ be a finite étale covering of the interior $U_{X}$ of $X$ which is tamely ramified over the generic points of $D_{X} \rightarrow X$. Let $Y$ be the normalization of $X$ in $U_{Y}$. Then the pair $\left(Y, U_{Y}\right)$ is toric (cf. Definition 1.2), hence defines a regular log scheme $(Y, N)$. Moreover, $U_{Y} \rightarrow U_{X}$ extends uniquely to a log étale morphism $(Y, N) \rightarrow(X, M)$.

This result is given as Theorem 3.3 in the text. It is the "log regular analogue" of the classical purity theorem which states that if $X$ is a regular scheme, and $U \subseteq X$ is an open subscheme such that the codimension in $X$ of $X-U$ is $\geq 2$, then any finite étale covering of $U$ extends (uniquely) to a finite étale covering of $X$. It was proven originally by K. Kato ([5]). Since, however, Kato's proof is not widely available in written form, and, moreover, this log purity result plays an essential role in the proof of Theorem A, we decided to give an independent treatment of Theorem B in the present paper. In fact, the author discovered Theorem B (independently of the work of Kato) in the course of trying to prove Theorem A, and only afterwards (as a result of communication with K. Fujiwara) did the author learn of the existence of Kato's proof. In fact, the proof of the present paper differs somewhat from that of $[5]$.

The proof of Theorem B that we give here runs as follows: We would like to reduce to the regular case, where one has the classical purity theorem. To do this, we blow up $(X, M)$ to form some $\left(X^{\prime}, M^{\prime}\right)$ for which $X^{\prime}$ is regular. Moreover, we show that the "relative $\pi_{1}$ " of the blow-up morphism $X^{\prime} \rightarrow X$ is trivial, so no information concerning coverings is lost by pulling back to $X^{\prime}$. This triviality of the relative $\pi_{1}$ is essentially an exercise in the combinatorics of toric varieties (Theorem 2.1). Now we would like to apply the classical purity theorem to the pull-back of the given covering to $X^{\prime}$. The problem (cf. the above discussion) is that since $X^{\prime}$ has new divisors, i.e., divisors that map to primes of codimension $\geq 2$ in $X$, it is no longer clear that the pulled-back covering over $X^{\prime}$ still satisfies the hypotheses of Theorem B, i.e., we do not know that the new covering is tamely ramified over the new divisors. To get around this problem, we construct (Proposition 2.2), for each new divisor $\mathfrak{p}^{\prime}$ of $X^{\prime}$, an intermediate blow-up $X^{\prime \prime} \rightarrow X$ such that $X^{\prime} \rightarrow X$ factors through $X^{\prime \prime}$ in a neighborhood of the given new divisor $\mathfrak{p}^{\prime}$. Moreover, the construction is such that if $\mathfrak{p}^{\prime}$ maps to a prime $\mathfrak{p}$ of height $h \geq 3$ in $X$, then it maps to a prime $\mathfrak{p}^{\prime \prime}$ of height $<h$ in $X^{\prime \prime}$. Also, the blow-up $X^{\prime \prime} \rightarrow X$ has the property that its fibers are of dimension $\leq 1$. In particular, divisors of $X^{\prime \prime}$ map to primes of $X$ of height $\leq 2$. Thus, by using induction on $h$ and applying the induction hypothesis to the prime $\mathfrak{p}^{\prime \prime}$ of $X^{\prime \prime}$, we see that we can reduce Theorem B to the case $h=2$. In dimension 2 , however, every regular log scheme is (étale locally) "isogenous" to one which arises from a divisor with normal crossings in a regular scheme (Corollary 1.8). Thus, we reduce to the regular case, where we can apply the classical purity theorem. This completes our summary of the proof of Theorem B.

Returning to the proof of Theorem A, once one has the log purity theorem, one can reduce Theorem A to the case where $h_{U_{X}}: U_{X} \rightarrow \overline{\mathcal{M}}$ admits a level $l$ structure (for some prime $l \geq 3$ - cf. Definition 5.6). In this case, $h_{U_{X}}$ automatically extends to the generic points of $D_{Y}$. Thus, applying the main result of [7] allows one to complete the proof of Theorem A. 
Once one has Theorem A, one can use it to compactify (cf. Theorem C below) hyperbolic polycurves, i.e., successive families of hyperbolic curves, as follows: Let $S$ be a scheme. A family of hyperbolic curves $U \rightarrow S$ over $S$ is a family of smooth compact genus $g$ curves with precisely $r$ points removed, for some nonnegative integers $g$ and $r$ such that $2 g-2+r \geq 1$ (see Definition 4.4 for a precise definition). A hyperbolic polycurve (cf. Definition 4.6) $U \rightarrow S$ is a morphism which factors as a composite of a finite number of morphisms, each of which is a family of hyperbolic curves.

The moduli stack $\mathcal{M}_{g, r}$ of ("split" - cf. Definition 5.2) hyperbolic curves is a smooth algebraic stack over $\mathbf{Z}$ which is not proper. To compactify it, it is necessary to introduce the notion of a stable curve (cf. Definition $4.3 ;[3,10]$ ). In this paper, we shall think of a family of stable curves as a pair $\left(X \rightarrow S, D_{M}\right)$, where $X \rightarrow S$ is a proper family of curves and $D_{M}$ (the "divisor of marked points") is a divisor in $X$ (such that $X \rightarrow S$ and $D$ satisfy certain properties). A stable polycurve (cf. Definition 4.5) is a pair $(X \rightarrow S, D)$ which, roughly speaking, factors as a composite of a finite number of families of stable curves. If $(X \rightarrow S, D)$ is a stable polycurve, then $X \rightarrow S$ is necessarily proper.

It is easy to see that if $(X \rightarrow S, D)$ is a stable polycurve, then if we let $U_{X} \stackrel{\text { def }}{=}$ $X-D$, and $U_{S}$ be the image of $U_{X}$ in $S$, then $U_{X} \rightarrow U_{S}$ is a hyperbolic polycurve. Thus, one may think of $(X \rightarrow S, D)$ as a sort of compactification of $U_{X} \rightarrow U_{S}$. Then the following result (Corollary 7.4 in the text) states that under certain natural conditions, such compactifications always exist and are functorial in $U_{X} \rightarrow U_{S}$ :

Theorem C. (Compactification Theorem) Let $\left(S, M_{S}\right)$ be a regular log scheme. Let $U_{S} \subseteq S$ be the interior of $S$. Let $U_{X} \rightarrow U_{S}$ be polyhyperbolic and saturated (cf. Definitions 4.6, 7.3). Then $U_{X} \rightarrow U_{S}$ may be compactified to a (log) polystable $\left(X, M_{X}\right) \rightarrow\left(S, M_{S}\right)$ (cf. Definition 4.5, 7.1).

Moreover, this compactification is natural in the following sense: Suppose that $\left(S^{\prime}, M_{S^{\prime}}\right)$ is a regular log scheme, and let $\left(X, M_{X}\right) \rightarrow\left(S, M_{S}\right)$, and $\left(X^{\prime}, M_{X^{\prime}}\right) \rightarrow\left(S^{\prime}, M_{S^{\prime}}\right)$ be $\log$ polystable with associated hyperbolic polycurves $U_{X} \rightarrow U_{S}$ and $U_{X^{\prime}} \rightarrow U_{S^{\prime}}$, respectively. Suppose, moreover, that we are given a commutative diagram:

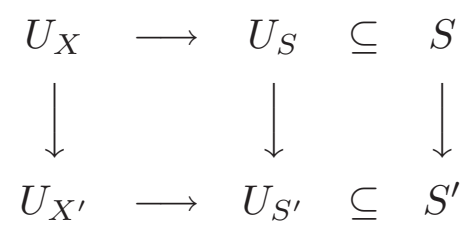

Then the square on the left "compactifies" uniquely to a commutative diagram:

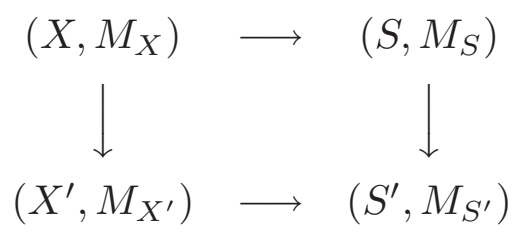


In particular, it makes sense to speak of "the" compactification of $U_{X} \rightarrow U_{S}$.

We remark that in an earlier version of this paper $([15])$, we proved a (weaker) version of Theorems $\mathrm{A}$ and $\mathrm{C}$ above, in the case where the toric pair $\left(S, U_{S}\right)$ is "polyregular," i.e., may be written as a composite of "locally stable morphisms" (cf. Definition 4.1) over a pair arising from a divisor with normal crossings in a regular scheme. Although this sort of treatment (i.e., as in [15]) of these results gives rise to theorems that are slightly weaker than those obtained in the present paper, it has the virtue of being independent of the theory of log schemes (cf. [8,9]), which is unfamiliar to some algebraic geometers. The main reasons why the polyregular case is easier to treat are as follows:

(1) Polyregular singularities are local complete intersections. Moreover, étale coverings and line bundles on dense open subsets of local complete intersection schemes tend to extend over the whole scheme as soon as they extend over the complement of a closed set of fairly small codimension (cf. the theory of [18]). This allows one (using certain results on the functoriality of the indeterminacy locus - cf., e.g., Lemma 5.2) to reduce the result to the case of polyregular singularities of dimension $\leq 3$.

(2) Polyregular singularities in dimension $\leq 3$ can be completely classified and are easy to understand. For instance, their singularities can be resolved explicitly. This means that one does not have to use such "black box-type" results as Theorem 2.1.

In $\S 1$, we review basic facts about regular log schemes. In $\S 2$, we analyze in some detail the structure of the sort of blow-ups that occur when one resolves toric singularities. This analysis is used to prove Theorem B (the "log purity theorem") in $\S 3$. In $\S 4$, we introduce various terminology concerning stable curves and successive families of stable curves. In $\S 5$, we discuss various "well-known" results concerning the extendability of morphisms defined on an open dense subset of a given normal scheme. In $\S 6$, we then explain how Theorem A follows from Theorem B and the main result of [7]. Finally, in $\S 7$, we observe that Theorem $\mathrm{C}$ is essentially a formal consequence of Theorem A.

Finally, I would like to thank Takeshi Tsuji for explaining to me various basic facts concerning regular log schemes, and Kazuhiro Fujiwara for informing me of the work of Prof. K. Kato.

\section{$\S 1$. Generalities on Regular Log Schemes}

Let $(X, M)$ be a fine log scheme (as in [8]), whose underlying scheme $X$ is noetherian. Let $\mathcal{O}_{X}^{\times} \subseteq \mathcal{O}_{X}$ denote the subsheaf of the structure sheaf of $X$ consisting of invertible sections. Thus, $M$ is a sheaf of abelian monoids in the étale topology of $X$, equipped 
with a morphism $\exp _{M}: M \rightarrow \mathcal{O}_{X}$ of étale sheaves of abelian monoids, and $\exp _{M}$ maps $\exp _{M}^{-1}\left(\mathcal{O}_{X}^{\times}\right)$isomorphically onto $\mathcal{O}_{X}^{\times} \subseteq \mathcal{O}_{X}$. (Note: In [8], the morphism $\exp _{M}$ is often denoted " $\alpha$. .") If $P$ is a abelian monoid, let us denote by $P^{\text {gp }}$ the abelian group associated to $P$. Clearly, this operation $P \mapsto P^{\text {gp }}$ of passing from abelian monoids to abelian groups is compatible with sheafification. Thus, let us denote by $M^{\text {gp }}$ the étale sheaf of abelian groups associated to $M$. Note that if $P$ is an integral monoid, then the natural map $P \rightarrow P^{g p}$ is injective. Since we have assumed that $(X, M)$ is fine (hence integral), it follows that for each $x \in X$, the stalk $\left\{M / \exp _{M}^{-1}\left(\mathcal{O}_{X}^{\times}\right)\right\}_{\bar{x}}$ (where $\bar{x}$ is the spectrum of the separable closure of the residue field of $x$ ) is an integral monoid.

An integral abelian monoid $P$ (written additively) is called saturated if for every $a \in P^{\text {gp }}$ such that $n \cdot a \in P$ for some positive integer $n$, it follows that $a \in P \subseteq P^{\text {gp }}$. The $\log$ scheme $(X, M)$ will be called saturated if, for each $x \in X,\left\{M / \exp _{M}^{-1}\left(\mathcal{O}_{X}^{\times}\right)\right\}_{\bar{x}}$ is saturated. If $x \in X$, let us write $I_{\bar{x}} \stackrel{\text { def }}{=} M_{\bar{x}} \backslash \mathcal{O}_{X, X / S}^{\times}$, and let us write $I_{\bar{x}} \cdot \mathcal{O}_{X, \bar{x}}$ for $\exp _{M}\left(I_{\bar{x}}\right) \cdot \mathcal{O}_{X, \bar{x}}$.

Let us henceforth assume that $(X, M)$ is saturated. Then, following [9], Definition 2.1, we make the following definition:

Definition 1.1. We shall say that $(X, M)$ is (log) regular at $x \in X$ if $\mathcal{O}_{X, \bar{x}} / I_{\bar{x}} \cdot \mathcal{O}_{X, \bar{x}}$ is regular, and, moreover, $\operatorname{dim}\left(\mathcal{O}_{X, \bar{x}}\right)=\operatorname{dim}\left(\mathcal{O}_{X, \bar{x}} / I_{\bar{x}} \cdot \mathcal{O}_{X, \bar{x}}\right)+\operatorname{rank}_{\mathbf{Z}}\left(M_{\bar{x}}^{\mathrm{gp}} / \exp _{M}^{-1}\left(\mathcal{O}_{X, \bar{x}}^{\times}\right)\right)$. We shall say that $(X, M)$ is $(\log )$ regular if $(X, M)$ is regular at every point of $X$.

Let us review some basic properties of $\log$ regular $(X, M)$ (cf. [9] for more details). If $(X, M)$ is $\log$ regular at a point $x \in X$, and $x$ lies in the closure of a point $y \in X$, then $(X, M)$ is $\log$ regular at $y$, as well ([9], Proposition 7.1). If $(Y, N) \rightarrow(X, M)$ is a $\log$ smooth morphism of saturated $\log$ schemes such that $X$ and $Y$ are noetherian, and $(X, M)$ is log regular, then $(Y, N)$ is also log regular ([9], Theorem 8.2). Suppose that $(X, M)$ is log regular. Then it follows that $X$ is normal and Cohen-Macaulay ([9], Theorem 4.1). Let $U \subseteq X$ be the open subscheme on which the $\log$ structure $M$ is trivial. We shall refer to this open subscheme as the interior of $X$. Let $D \subseteq X$ be the complement of $U$, with the reduced induced scheme structure. (When there are several log schemes involved, we shall write $U_{X}, D_{X}, M_{X}$, for $U, D$, and $M$ to show that they are the respective objects associated to $(X, M)$.) Then $D$ is a divisor in $X$, and the morphism $\exp _{M}: M \rightarrow \mathcal{O}_{X}$ is isomorphic (cf. [9], Theorem 11.6) to the natural morphism $\left(\mathcal{O}_{X} \cap i_{*} \mathcal{O}_{U}^{\times}\right) \hookrightarrow \mathcal{O}_{X}$, where $i: U \hookrightarrow X$ is the natural inclusion, and " $\mathcal{O}_{X} \bigcap i_{*} \mathcal{O}_{U}^{\times}$" denotes the subsheaf of $\mathcal{O}_{X}$ consisting of regular functions on $X$ whose restriction to $U$ is invertible. In particular, $\exp _{M}$ is necessarily injective. If $f$ is a section of $\mathcal{O}_{X}$ that lies in the image of $\exp _{M}$, then we shall write $\log _{M}(f)$ for the unique section of $M$ that maps under $\exp _{M}$ to $f$. Thus, it follows that whether or not $(X, M)$ is regular is an intrinsic property of the pair $(X, U)$. In particular, it is meaningful to make the following definition:

Definition 1.2. If $X$ is a normal scheme, and $U \subseteq X$ is an open subscheme of $X$, then we shall call $(X, U)$ a toric pair if $(X, U)$ arises as above from some log regular log 
structure on $X$. If $(X, U)$ is a toric pair, then we shall refer to the singularities of $X$ as toric singularities.

In this paper, we shall say that a closed subscheme $D$ in a regular noetherian scheme $X$ is a "divisor with normal crossings" if $D$ is a divisor which, étale locally on $X$, is (scheme-theoretically) defined by a product $t_{1} \cdot t_{2} \cdot \ldots \cdot t_{r}$, where $t_{1}, \ldots, t_{r}$ is part of a regular system of parameters. Thus, if $D$ is a divisor with normal crossings in $X$, then $(X, U)$ (where $U \stackrel{\text { def }}{=} X-D$ ) forms a toric pair. In other words, one may think of the notion of a toric pair as a sort of generalization of the notion of a divisor with normal crossings.

Now let us suppose that $(X, M)$ is log regular and that $X$ is strictly henselian with unique closed point $x \in X$. Let $P \stackrel{\text { def }}{=} M_{x} / \log _{M}\left(\mathcal{O}_{X, x}^{\times}\right)$. Thus, $P$ is a finitely generated, integral, saturated monoid. Moreover, $P$ does not contain any nonzero invertible elements, i.e., $a,-a \in P$ implies $a=0$. In particular, $P$ (and hence also $P^{g p}$ ) is torsion-free. Thus, $P^{\text {gp }}$ is a finitely generated free abelian group. Moreover, we have an exact sequence of abelian groups

$$
0 \rightarrow \log _{M}\left(\mathcal{O}_{X, x}^{\times}\right)\left(\cong \mathcal{O}_{X, x}^{\times}\right) \rightarrow M^{g p} \rightarrow P^{g p} \rightarrow 0
$$

Since $P^{g p}$ is free, it follows that this exact sequence always splits.

Definition 1.3. We shall refer to a splitting $P^{g p} \rightarrow M^{g p}$ of the above exact sequence as a clean chart at $x$.

Note that we also have an exact sequence of abelian monoids:

$$
0 \rightarrow \log _{M}\left(\mathcal{O}_{X, x}^{\times}\right) \rightarrow M \rightarrow P \rightarrow 0
$$

Moreover, it is clear that this exact sequence may be identified with the pull-back of the preceding exact sequence via $P \rightarrow P^{\text {gp }}$. Thus, a clean chart induces a splitting $P \rightarrow M$. Note that such a splitting $P \rightarrow M$ defines a chart (in the sense of [8]) for the log scheme $(X, M)$. If a clean chart has been fixed, then we shall write $\exp _{M}(a)$ (where $a \in P^{\mathrm{gp}}$ ) for the (rational) section of $\mathcal{O}_{X}$ defined by applying to $a$ the composite of the splitting $P^{\mathrm{gp}} \rightarrow M^{\mathrm{gp}}$ with (the morphism induces on (-) ${ }^{\mathrm{gp}}$ 's by) $\exp _{M}: M \rightarrow \mathcal{O}_{X}$.

Let $n$ be a positive integer. Then we would like to define a morphism

$$
(Y, N) \rightarrow(X, M)
$$

of $\log$ schemes as follows: Let us write $\mathbf{Z}[P]$ for the monoid ring of $P$ over $\mathbf{Z}$. Let $n_{P}$ : $\mathbf{Z}[P] \rightarrow \mathbf{Z}[P]$ denote the $\mathbf{Z}$-algebra morphism induced the "multiplication by $n$ " on $P$. Let 
$Y \stackrel{\text { def }}{=} X \times_{\mathbf{Z}[P], n_{P}} \mathbf{Z}[P]$, and let $N$ be the $\log$ structure associated to the chart $P \rightarrow \mathcal{O}_{Y}$ (where this " $P$ " is the $P$ in the range of the morphism $n_{P}$ ).

Lemma 1.4. $(Y, N)$ is log regular. Moreover, the natural morphism $Y \rightarrow X$ is finite over $X$, and flat of degree $n^{\operatorname{rankz}\left(P^{\text {gp }}\right)}$ over $U_{X}$.

Proof. The last sentence is immediate from the definition of $(Y, N)$. Thus, it suffices to prove that $(Y, N)$ is $\log$ regular. Since $\log$ regularity is stable under genericization ([9], Proposition 7.1), it suffices to prove that $(Y, N)$ is log regular at the unique point $y \in Y$ lying over $x \in X$. Since it follows immediately from the definitions that $\mathcal{O}_{Y, y} / I_{y} \cdot \mathcal{O}_{Y, y}=$ $\mathcal{O}_{X, x} / I_{x} \cdot \mathcal{O}_{X, x}$, we thus see that we are in a situation where we can apply Theorem 6.1 of [9]. One thus concludes as in the last paragraph of the proof of Theorem 8.2 of [9], p. 1087.

Definition 1.5. We shall refer to $(Y, N) \rightarrow(X, M)$ as the dilation of magnitude $n$ associated to the clean chart $P^{\mathrm{gp}} \rightarrow M^{\mathrm{gp}}$. If $(Y, N) \rightarrow(X, M)$ is a dilation, then we shall refer to $(Y, N)$ as a dilate of $(X, M)$.

Now let $\mathfrak{p} \in X$ be a point of $X$. Let us write $X_{\overline{\mathfrak{p}}}$ for the strict henselization of $X$ at $\mathfrak{p}$. Note that the clean chart $P^{\mathrm{gp}} \rightarrow M^{\mathrm{gp}}$ defines a morphism $\left.P^{\mathrm{gp}} \rightarrow M^{\mathrm{gp}} \rightarrow M^{\mathrm{gp}}\right|_{X_{\overline{\mathfrak{p}}}}$ such that

$$
\left.P^{\mathrm{gp}} \rightarrow\left(M^{\mathrm{gp}} /\left(\log _{M}\left(\mathcal{O}_{X}^{\times}\right)\right)\right)\right|_{X_{\overline{\mathfrak{p}}}}
$$

is surjective. In particular, there exists a finitely generated, free $\mathbf{Z}$-module $P_{\mathfrak{p}}^{\mathrm{gp}} \subseteq P^{\mathrm{gp}}$ such that the restriction

$$
\left.P_{\mathfrak{p}}^{\mathrm{gp}} \rightarrow M^{\mathrm{gp}}\right|_{\overline{\bar{p}}}
$$

of $\left.P^{\mathrm{gp}} \rightarrow M^{\mathrm{gp}}\right|_{X_{\overline{\mathfrak{p}}}}$ to $P_{\mathfrak{p}}^{\mathrm{gp}}$ defines a clean chart for $X_{\overline{\mathfrak{p}}}$. Since dilations of $X_{\overline{\mathfrak{p}}}$ with respect to this clean chart are obtained by forming the normalization of $X_{\overline{\mathfrak{p}}}$ in the finite, flat covering of $U_{\overline{\mathfrak{p}}}$ obtained by extracting roots of elements of the image of $P_{\mathfrak{p}}^{\text {gp }}$ in $\mathcal{O}_{U_{\overline{\mathfrak{p}}}}$, and such elements may also be obtained as elements of the image under the natural morphism $\mathcal{O}_{U} \rightarrow \mathcal{O}_{U_{\overline{\mathfrak{p}}}}$ of elements of $\exp _{M}\left(P^{\mathrm{gp}}\right)$, it thus follows that:

Proposition 1.6. For any $\mathfrak{p} \in X$, there exists a clean chart for $X_{\overline{\mathfrak{p}}}$ such that any dilation of $X_{\overline{\mathfrak{p}}}$ with respect to this clean chart is dominated by the restriction to $X_{\overline{\mathfrak{p}}}$ of a dilation with respect to the given clean chart $P^{\mathrm{gp}} \rightarrow M^{\mathrm{gp}}$ of $X$. 
Finally, before continuing, we pause to consider the structure of $P$ in greater detail in the case $\operatorname{rank}_{\mathbf{Z}}\left(P^{\mathrm{gp}}\right) \leq 2$.

Proposition 1.7. If $\operatorname{rank}_{\mathbf{Z}}\left(P^{\mathrm{gp}}\right)=1$, then $P \cong \mathbf{N} \stackrel{\text { def }}{=}\{a \in \mathbf{Z} \mid a \geq 0\}$. If $\operatorname{rank}_{\mathbf{Z}}\left(P^{\mathrm{gp}}\right)=$ 2, then there exist $a, b \in P_{\mathbf{Q}}^{\mathrm{gp}} \stackrel{\text { def }}{=} P^{\mathrm{gp}} \otimes_{\mathbf{z}} \mathbf{Q}$ such that: (i) $P \subseteq \mathbf{N} \cdot a+\mathbf{N} \cdot b$; (ii) there exists a positive integer $l$ such that $l \cdot a, l \cdot b \in P$.

Proof. The rank 1 case is trivial. For a discussion of the rank 2 case, we refer to the discussion preceding Proposition 1.19 of $\S 1.7$ of [17]. Indeed, in the notation of loc. cit., if $\alpha, \beta \in P$ are the primitive elements denoted " $n, n^{\prime}$ " (in the third paragraph of p. 24 of $[17])$, then it suffices to take $a \stackrel{\text { def }}{=} \alpha / l, b \stackrel{\text { def }}{=} \beta / l$ for some appropriate $l$.

Corollary 1.8. If $\operatorname{rank}_{\mathbf{Z}}\left(P^{\mathrm{gp}}\right)=1$, then $X$ is regular and $D_{X}$ is a regular divisor in $X$. If $\operatorname{rank}_{\mathbf{Z}}\left(P^{\mathrm{gp}}\right)=2$, then there exists a positive integer $n$ such that any dilation $(Y, N) \rightarrow(X, M)$ of magnitude divisible by $n$ factors through some $\left(Y^{\prime}, M^{\prime}\right)$ such that: (i) $Y^{\prime}$ is regular, and $D_{Y^{\prime}}$ is a divisor with normal crossings in $Y^{\prime}$; (ii) the morphisms $Y \rightarrow Y^{\prime}$ and $Y^{\prime} \rightarrow X$ are finite (over $X$ ) and flat over $U_{X}$.

Proof. The rank 1 case is trivial. The rank 2 case follows from Proposition 1.7 by letting $(Y, N) \rightarrow(X, M)$ be the dilation of magnitude $l$, and letting $\left(Y^{\prime}, N^{\prime}\right) \rightarrow(X, M)$ (respectively, $\left.(Y, N) \rightarrow\left(Y^{\prime}, N^{\prime}\right)\right)$ be the morphism defined by $\mathbf{Z}[P] \hookrightarrow \mathbf{Z}[\mathbf{N} \cdot a+\mathbf{N} \cdot b]$ (respectively, $\mathbf{Z}[\mathbf{N} \cdot a+\mathbf{N} \cdot b] \hookrightarrow \mathbf{Z}\left[\frac{1}{l} \cdot P\right]$ ). Note that the $\log$ regularity of $\left(Y^{\prime}, N^{\prime}\right)$ follows by the same argument as that used in the proof of Lemma 1.4 to prove the log regularity of $(Y, N)$.

Remark. The property discussed in Corollary 1.8, i.e., that "up to isogeny" every toric pair is "regular" (i.e., arises from a divisor with normal crossings in a regular scheme) is false in dimensions $\geq 3$. Indeed, if it were true, then it would follow that if $(X, M)$ is $\log$ regular, then $X$ is necessarily locally $\mathbf{Q}$-factorial, i.e., some nonzero multiple of every Weil divisor on $X$ is Cartier. On the other hand, an easy calculation (involving blowing up at the origin $u=v=x=y=0$ ) reveals that the pair

$$
X \stackrel{\text { def }}{=} \operatorname{Spec}(k[[u, v, x, y]] /(u v-x y)) \supseteq U \stackrel{\text { def }}{=} X\left[\frac{1}{u}, \frac{1}{v}\right]
$$

is toric, but not locally $\mathbf{Q}$-factorial. 


\section{$\S 2$. Blow-ups of Regular Log Schemes}

We continue with the notation of $\S 1$. Thus, we assume that $(X, M)$ is log regular and that $X$ is strictly henselian with unique closed point $x \in X$. In fact, in this $\S$, we would also like to assume that $X$ is the spectrum of a complete local ring.

Let us recall the apparatus of "fans," as discussed for instance in [17], p. 2. Let $r \stackrel{\text { def }}{=} \operatorname{rank}_{\mathbf{Z}}\left(P^{\mathrm{gp}}\right)$. Then one may think of the finitely generated saturated monoid $P$ as being defined (cf. [17], Proposition 1.1, p. 3) by a strongly convex rational polyhedral cone $\sigma_{P} \subseteq \mathbf{R}^{r}$. More generally, a fan is a nonempty collection of strongly convex rational polyhedral cones in $\mathbf{R}^{r}$ satisfying certain conditions (cf. [17], p. 2). If $\Delta$ is the fan arising from a locally finite nonsingular subdivision of $\sigma_{P}$ (cf. [17], p. 23, Corollary 1.18), then $\Delta$ defines a proper, birational morphism

$$
f^{\log }:(Y, N) \rightarrow(X, M)
$$

(cf. [9], §10) such that $(Y, N)$ is $\log$ regular; $Y$ is regular; and $D_{Y} \subseteq Y$ is a divisor with normal crossings in $Y$. It is known that such a $\Delta$ always exists (cf. [17], §1.5).

Let $Z \subseteq Y$ be an irreducible component of $\left(Y_{x}\right)_{\text {red }}$ (where $Y_{x} \stackrel{\text { def }}{=} f^{-1}(x)$ ). Then the ideal defining the closed subscheme $Z \subseteq Y$ is (Zariski) locally generated by sections of $\mathcal{O}_{Y}$ of the form $\exp _{N}(a)$, for $a \in P^{\text {gp }}$. In particular, it follows immediately that $Z$ is regular. Let us define the closed subscheme $D_{Z} \subseteq Z$ Zariski locally on $Z$ as the restriction to $Z$ of all the irreducible components of $D_{Y}$ that do not contain $Z$. Let $U_{Z} \stackrel{\text { def }}{=} Z-D_{Z}$. Then I claim that $Z$ is a toric variety whose torus embedding is $U_{Z} \subseteq Z$. Indeed, this follows immediately from the fact that, Zariski locally, $Z$ is, by construction, the spectrum of the monoid ring associated to some quotient of a submonoid of $P^{\mathrm{gp}}$. Thus, in particular, $U_{Z}$ is isomorphic to some product of $\mathbf{G}_{m}$ 's over $k \stackrel{\text { def }}{=} k(x)$, the residue field of $x \in X$. Let us write $\widehat{Y}$ for the formal scheme obtained by completing $Y$ along the proper $k$-scheme $Y_{x}$.

Next, recall that there is a certain correspondence between certain portions of $\Delta$ or $\sigma_{P}$ and various subobjects of $Y$. For instance, each of the cones $\sigma \in \Delta$ corresponds to affine opens $U_{\sigma} \subseteq Y, \widehat{U}_{\sigma} \subseteq \widehat{Y}$ (cf. Propositions 1.3, 1.4 of [17], p. 7). Under this correspondence, the cones $\sigma \in \Delta$ that do not intersect the interior $\operatorname{Int}\left(\sigma_{P}\right)$ of $\sigma_{P}$ correspond to the $U_{\sigma} \subseteq Y \backslash Y_{x}$, while the cones $\sigma \in \Delta$ that intersect $\operatorname{Int}\left(\sigma_{P}\right)$ correspond to the $U_{\sigma}$ that intersect the fiber $Y_{x}$. Let $\sigma \in \Delta$ be an $r$-dimensional (i.e., top dimensional) cone in $\Delta$. Thus, since $\sigma$ is nonsingular (i.e., abstractly isomorphic to $\mathbf{N}^{r}$ ), it follows that $\widehat{U}_{\sigma}$ is the completion of $Y$ along some subscheme $W_{\sigma} \subseteq\left(Y_{x}\right)_{\text {red }} \subseteq Y$, where $W_{\sigma}$ may be identified with a closed, reduced subscheme of $\mathbf{A}_{k}^{r}$ (i.e., $r$-dimensional affine space over $k$ ) which is the union of a finite number of linear subspaces (passing through the origin) of $\mathbf{A}_{k}^{r}$. Note that each of these linear subspaces is an open dense subset of some $Z \subseteq Y$ of the sort considered in the preceding paragraph.

We would like to consider the algebraic fundamental group $\pi_{1}(\widehat{Y})$ (for some choice of basepoint in $Y_{x}$, which, for convenience, we omit in the following) of $\widehat{Y}$. Since the étale site 
is invariant under nilpotent thickenings, it follows that $\pi_{1}(\widehat{Y})=\pi_{1}\left(Y_{x}\right)=\pi_{1}\left(\left(Y_{x}\right)_{\text {red }}\right)$. Let $Z \subseteq\left(Y_{x}\right)_{\text {red }}$ be an irreducible component of $\left(Y_{x}\right)_{\text {red }}$. Then $Z$ is a smooth, proper, rational variety over $k$. In particular, its fundamental group $\pi_{1}(Z)$ is trivial. (Indeed, since $Z$ is a proper, toric variety, it lifts naturally to a toric variety in characteristic zero. Moreover, since $\pi_{1}(Z)$ is a quotient of this toric variety in characteristic zero, it suffices to observe that the fundamental group of any smooth, proper toric variety in characteristic zero is trivial - but this follows, for instance, from Proposition 1.9 of [17], p. 14.) Now let $\widehat{Y}^{\prime} \rightarrow \widehat{Y}$ be an arbitrary finite étale covering. Since the restriction of this covering to any $Z$ is trivial, it follows that the restriction of this covering to each of the irreducible components of any $W_{\sigma}$ (as in the preceding paragraph) is trivial. But since all the irreducible components of $W_{\sigma}$ pass through the origin in $\mathbf{A}_{k}^{r}$, it follows that the restriction of $\widehat{Y}^{\prime} \rightarrow \widehat{Y}$ to $W_{\sigma}$ is trivial.

More generally, for any $\sigma \in \Delta$ such that $\sigma \bigcap \operatorname{Int}\left(\sigma_{P}\right) \neq \emptyset$, let $W_{\sigma} \stackrel{\text { def }}{=} \widehat{U}_{\sigma} \bigcap\left(Y_{x}\right)_{\text {red }}$. Since any such $\sigma$ is a face of an $r$-dimensional cone $\sigma^{\prime} \in \Delta$ (so $W_{\sigma} \subseteq W_{\sigma^{\prime}}$ ), it follows that the restriction of $\widehat{Y}^{\prime} \rightarrow \widehat{Y}$ to any such $W_{\sigma}$ is trivial. Thus, for any such $\sigma$, we obtain a (trivial) finite étale covering $W_{\sigma}^{\prime} \rightarrow W_{\sigma}$. Since these étale coverings are compatible with respect to restriction from $W_{\sigma}$ to $W_{\tau}$ (where $\tau$ is a face of $\sigma$ ), it follows that we may construct a finite étale covering $\mathcal{C} \rightarrow \operatorname{Int}\left(\sigma_{P}\right)$ as follows: For each $\sigma \in \Delta$ as above, we let $\mathcal{C}_{\sigma} \rightarrow \sigma \bigcap \operatorname{Int}\left(\sigma_{P}\right)$ be the trivial finite étale covering whose set of connected components is equal to the set of connected components of $W_{\sigma}^{\prime}$. Then because of the compatibility of the $W_{\sigma}^{\prime} \rightarrow W_{\sigma}$ with respect to restriction, it follows that the $\mathcal{C}_{\sigma} \rightarrow \sigma \bigcap \operatorname{Int}\left(\sigma_{P}\right)$ glue together to form a finite étale covering $\mathcal{C} \rightarrow \operatorname{Int}\left(\sigma_{P}\right)$ of the topological space $\operatorname{Int}\left(\sigma_{P}\right)$. On the other hand, since $\sigma_{P}$ is a convex cone, it is clear that $\operatorname{Int}\left(\sigma_{P}\right)$ is simply connected. Thus, the covering $\mathcal{C} \rightarrow \operatorname{Int}\left(\sigma_{P}\right)$ splits. Since the covering $\widehat{Y}^{\prime} \rightarrow \widehat{Y}$ is defined "by the same combinatorics" as $\mathcal{C} \rightarrow \operatorname{Int}\left(\sigma_{P}\right)$, it thus follows that $\widehat{Y}^{\prime} \rightarrow \widehat{Y}$ splits, i.e., that $\pi_{1}(\widehat{Y})$ is trivial. Since $f: Y \rightarrow X$ is proper, it follows from the "theorem on formal functions" (see, e.g., Theorem 11.1 of Chapter III of [11]) that $\pi_{1}(Y)$ is trivial.

We summarize the above discussion in the following:

Theorem 2.1. There exists a proper, birational morphism of finite type $f^{\log }:(Y, N) \rightarrow$ $(X, M)$, where $(Y, N)$ is log regular; $Y$ is regular; and $D_{Y}$ is a divisor with normal crossings in $Y$. Moreover, this morphism may be chosen so that any irreducible component $Z$ of $f^{-1}(x)_{\text {red }}$ is a smooth, proper toric variety such that the torus inside $Z$ is given as follows: Let $D_{Z} \subseteq Z$ be the closed subscheme defined locally on $Z$ as the restriction to $Z$ of all the irreducible components of $D_{Y}$ that do not contain $Z$. Then the torus inside $Z$ is given by $U_{Z} \stackrel{\text { def }}{=} Z-D_{Z}$. In particular, $U_{Z}$ is isomorphic to a product of $\mathbf{G}_{m}$ 's over $k \stackrel{\text { def }}{=} k(x)$ (the residue field of $x \in X)$. Finally, the fundamental group $\pi_{1}(Y)$ is trivial.

In this remainder of this $\S$, we would like to analyze the structure of $f^{\log }:(Y, N) \rightarrow$ $(X, M)$ at the generic points of $D_{Y}$ in greater detail. Let $\mathfrak{p}_{Y} \in Y$ be a generic point of $D_{Y}$ that maps to $x \in X$. Then $\mathfrak{p}_{Y}$ defines a "valuation"

$$
v: P \rightarrow \mathbf{N}
$$


on $P$ by letting $v(a)$ (for $a \in P$ ) be the order at $\mathfrak{p}_{Y}$ of $\exp _{N}(a)$. Let $k\left(\mathfrak{p}_{Y}\right)$ be the residue field of $\mathfrak{p}_{Y}$. Thus, $k\left(\mathfrak{p}_{Y}\right)$ is a finitely generated field extension of $k \stackrel{\text { def }}{=} k(x)$ which is generated (as a field extension of $k$ ) by the images of $\exp _{N}(a-b)$ in $k\left(\mathfrak{p}_{Y}\right)$, where $a, b \in P$ range over all pairs of elements such that $v(a)=v(b)$. Now suppose that the height ht $\mathrm{X}_{X}(x)$ of $x$ (as point of $X$ ) is $\geq 2$. Since $X$ is the spectrum of complete local ring, it follows that $X$ is universally catenary, hence that the transcendence degree of $k\left(\mathfrak{p}_{Y}\right)$ over $k$ is $\geq 1$ (cf. [13], §14, 34). Thus, there exist $a, b \in P$ such that $v(a)=v(b)$, and, moreover, the image of $\exp _{N}(a-b)$ in $k\left(\mathfrak{p}_{Y}\right)$ is transcendental over $k$. Note that since $v(c)>0$ for all nonzero $c \in P$, it follows (from the fact that $v(a-b)=0$ ) that $a-b \notin P$. Also, there does not exist a $c \in P$ such that $a$ and $b$ are both multiples of $c$. (For if this were the case, then $\exp _{N}(a-b)=1$, which is not transcendental over $k$.) Let us write $R$ for the submonoid of $P^{\text {gp }}$ generated by $P$ and $a-b \neq 0$, and $R^{\prime}$ for the smallest saturated submonoid of $P^{g p}$ containing $R$. Thus, $R^{\prime}$ is a finitely generated saturated integral monoid.

Let $Z \stackrel{\text { def }}{=} X\left[\exp _{M}(a) \cdot\left(\exp _{M}(b)\right)^{-1}\right]$ (i.e., the affine scheme birational to $X$ which is obtained from $X$ by adjoining the rational function in brackets). Let $\left(Z^{\prime}, Q^{\prime}\right)$ be the $\log$ scheme given as follows: $Z^{\prime} \stackrel{\text { def }}{=} X \otimes_{\mathbf{Z}[P]} \mathbf{Z}\left[R^{\prime}\right] ; Q^{\prime}$ is the log structure defined by the chart given by the natural morphism $R^{\prime} \rightarrow \mathcal{O}_{Z^{\prime}}$. Thus, we have a natural morphism $\left(Z^{\prime}, Q^{\prime}\right) \rightarrow$ $(X, M)$ of saturated $\log$ schemes such that $Z^{\prime} \rightarrow X$ factors through $Z$. Moreover, it is clear that this morphism is log étale (hence log smooth). Thus, it follows (by [9], Theorem 8.2) that $\left(Z^{\prime}, Q^{\prime}\right)$ is $\log$ regular. Moreover, it is immediate that the fibers of $Z \rightarrow X$ and $Z^{\prime} \rightarrow X$ have dimension $\leq 1$, and that $Z^{\prime} \rightarrow Z$ is finite. Let $Z_{x}^{\prime}$ (respectively, $Z_{x}$ ) be the fiber of $Z^{\prime} \rightarrow X$ (respectively, $Z \rightarrow X$ ) over $x \in X$. Then $Z_{x}^{\prime} \rightarrow Z_{x}$ is finite, and $Z_{x}=\operatorname{Spec}(k[t])$, where $t$ is the indeterminate over $k$ which is the image of $\exp _{N}(a-b)$. Let us write $\mathfrak{p}_{Z} \in Z$ for the generic point of $Z_{x}$. Thus, (since $X$ is universally catenary) $\operatorname{ht}_{Z}\left(\mathfrak{p}_{Z}\right)=\operatorname{ht}_{X}(x)-1 \geq 1$.

Now let $\left(Y^{\prime}, N^{\prime}\right)$ denote the Zariski localization of $(Y, N)$ at $\mathfrak{p}_{Y}$. Thus, we have a natural localization morphism $\left(Y^{\prime}, N^{\prime}\right) \rightarrow(Y, N)$. Composing this morphism with $f^{\log }$ gives us a morphism $\left(Y^{\prime}, N^{\prime}\right) \rightarrow(X, M)$. Moreover, it is clear from the definition of $P^{\prime}$ that this morphism $\left(Y^{\prime}, N^{\prime}\right) \rightarrow(X, M)$ factors through $\left(Z^{\prime}, Q^{\prime}\right)$. Thus, we get a morphism $Y^{\prime} \rightarrow Z^{\prime} \rightarrow Z$. Let us write $\mathfrak{p}_{Y^{\prime}} \in Y^{\prime}$ for the point induced by $\mathfrak{p}_{Y}$. Now I claim that the image in $Z$ of $\mathfrak{p}_{Y^{\prime}}$ is $\mathfrak{p}_{Z} \in Z$. Indeed, since $\mathfrak{p}_{Y^{\prime}} \in Y^{\prime}$ maps to $x \in X$, it follows that the image in $Z$ of $\mathfrak{p}_{Y^{\prime}}$ lies in $Z_{x}$. On the other hand, since the image of " $t$ " (i.e., the indeterminate such that $\left.Z_{x}=\operatorname{Spec}(k[t])\right)$ in $k\left(\mathfrak{p}_{Y^{\prime}}\right)=k\left(\mathfrak{p}_{Y}\right)$ is, by construction, transcendental over $k$, it thus follows that the image in $Z$ of $\mathfrak{p}_{Y^{\prime}}$ must be the generic point of $Z_{x}$, i.e., $\mathfrak{p}_{Z}$. This completes the proof of the claim. Let $\mathfrak{p}_{Z^{\prime}} \in Z^{\prime}$ be the image in $Z^{\prime}$ of $\mathfrak{p}_{Y^{\prime}}$. Thus, $\operatorname{ht}_{Z^{\prime}}\left(\mathfrak{p}_{Z^{\prime}}\right)=\operatorname{ht}_{Z}\left(\mathfrak{p}_{Z}\right)=\operatorname{ht}_{X}(x)-1$.

Next, let us denote by $Z_{\overline{\mathfrak{p}}_{Z^{\prime}}}^{\prime}$ the strict henselization of $Z^{\prime}$ at $\mathfrak{p}_{Z^{\prime}}$. Observe that the original clean chart $P^{\mathrm{gp}} \rightarrow M^{\mathrm{gp}}$ defines a morphism $P^{\mathrm{gp}} \rightarrow\left(Q^{\prime}\right)^{\mathrm{gp}}$ such that $P^{\mathrm{gp}} \rightarrow$

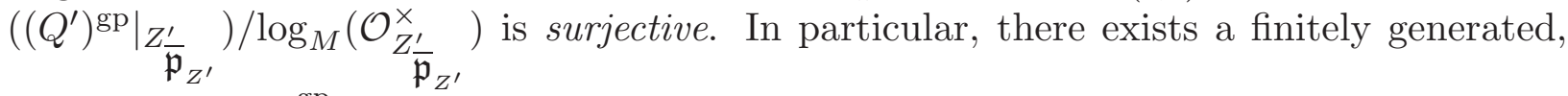
free $\mathbf{Z}$-module $P_{\mathfrak{p}_{Z^{\prime}}}^{\mathrm{gp}} \subseteq P^{\mathrm{gp}}$ such that the restriction 


$$
\left.P_{\mathfrak{p}_{Z^{\prime}}}^{\mathrm{gp}} \rightarrow\left(Q^{\prime}\right)^{\mathrm{gp}}\right|_{Z_{\overline{\mathfrak{p}}_{Z^{\prime}}^{\prime}}}
$$

of $\left.P^{\mathrm{gp}} \rightarrow\left(Q^{\prime}\right)^{\mathrm{gp}}\right|_{Z_{\overline{\mathfrak{p}}_{Z^{\prime}}}^{\prime}}$ to $P_{\mathfrak{p}_{Z^{\prime}}}^{\mathrm{gp}}$ defines a clean chart for $Z_{\overline{\mathfrak{p}}_{Z^{\prime}}}^{\prime}$. Since dilations of $Z_{\overline{\mathfrak{p}}_{Z^{\prime}}^{\prime}}$ with respect to this clean chart are obtained by forming the normalization of $Z_{\overline{\mathfrak{p}}_{Z^{\prime}}}^{\prime}$ in the finite, flat covering of $U_{Z_{\overline{\mathfrak{p}}_{Z^{\prime}}}}$ obtained by extracting roots of elements of the image of $P_{\mathfrak{p}_{Z^{\prime}}}^{\mathrm{gp}}$ in $\mathcal{O}_{U_{Z_{\bar{p}^{\prime}}}}$, and such elements may also be obtained as elements of the image under the

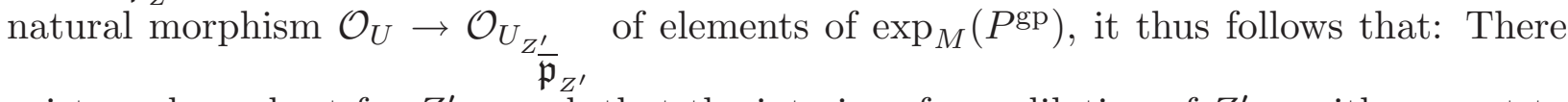
exists a clean chart for $Z_{\overline{\mathfrak{p}}_{Z^{\prime}}}^{\prime}$ such that the interior of any dilation of $Z_{\overline{\mathfrak{p}}_{Z^{\prime}}}^{\prime}$ with respect to this clean chart is dominated by the restriction to $Z_{\overline{\mathfrak{p}}_{Z^{\prime}}}^{\prime}$ of the interior of a dilation with respect to the given clean chart $P^{\mathrm{gp}} \rightarrow M^{\mathrm{gp}}$ of $X$.

We summarize the above discussion as follows:

Proposition 2.2. Let $(Y, N) \rightarrow(X, M)$ be as in Theorem 2.1. Let $\mathfrak{p}_{Y} \in Y$ be a generic point of $D_{Y}$ that maps to $x \in X$. Let $\left(Y^{\prime}, N^{\prime}\right)$ be the Zariski localization of $(Y, N)$ at $\mathfrak{p}_{Y}$, and write $\mathfrak{p}_{Y^{\prime}} \in Y^{\prime}$ for the point of $Y^{\prime}$ that corresponds to $\mathfrak{p}_{Y} \in Y$. Suppose that the height of $x \in X$ is $\geq 2$. Then the morphism $\left(Y^{\prime}, N^{\prime}\right) \rightarrow(X, M)$ factors as a composite of two morphisms $\left(Y^{\prime}, N^{\prime}\right) \rightarrow\left(Z^{\prime}, Q^{\prime}\right) \rightarrow(X, M)$ such that:

(1) $\left(Z^{\prime}, Q^{\prime}\right)$ is log regular;

(2) the morphisms $Y^{\prime} \rightarrow Z^{\prime}$ and $Z^{\prime} \rightarrow X$ are birational; in fact, $Z^{\prime} \rightarrow X$ is an isomorphism over the interior $U_{X}$ of $X$;

(3) the fibers of $Z^{\prime} \rightarrow X$ have dimension $\leq 1$ (thus, in particular, any point of $Z^{\prime}$ of height 1 necessarily maps to a point of height $\leq 2$ in $\left.X\right)$;

(4) the image $\mathfrak{p}_{Z^{\prime}} \in Z^{\prime}$ of $\mathfrak{p}_{Y^{\prime}} \in Y^{\prime}$ satisfies $\mathrm{ht}_{Z^{\prime}}\left(\mathfrak{p}_{Z^{\prime}}\right)=\mathrm{ht}_{X}(x)-1$.

Moreover, if we write $Z_{\overline{\mathfrak{p}}_{Z^{\prime}}}^{\prime}$ for the strict henselization of $Z^{\prime}$ at $\mathfrak{p}_{Z^{\prime}}$, then there exists a clean chart for $Z_{\overline{\mathfrak{p}}_{Z^{\prime}}}^{\prime}$ such that the interior of any dilation of $Z_{\overline{\mathfrak{p}}_{Z^{\prime}}}^{\prime}$ with respect to this clean chart is dominated by the restriction to $U_{Z_{\overline{\mathfrak{p}}_{Z^{\prime}}^{\prime}}}$ of the interior of a dilation with respect to the given clean chart $P^{\mathrm{gp}} \rightarrow M^{\mathrm{gp}}$ of $X$. 


\section{§. Log Purity}

In this $\S$, we give a proof of an unpublished result due originally to K. Kato which is a "log regular analogue" of the well-known classical purity theorem ([18], Exposé X, p. 118, Théorème 3.4) on extending étale covers over regular schemes.

Lemma 3.1. Let $(X, M)$ be a regular log scheme. Let $V \rightarrow U_{X}$ be a finite étale covering of the interior $U_{X}$ of $X$ which is tamely ramified over the generic points of the divisor $D_{X} \subseteq X$. Suppose that $X$ is strictly henselian with unique closed point $x \in X$. Then there exists a positive integer $n$ such that if $\left(Y^{\prime}, N^{\prime}\right) \rightarrow(X, M)$ is any dilation (cf. Definition 1.5) of magnitude divisible by $n$, then the pull-back of the covering $V \rightarrow U_{X}$ to $U_{Y^{\prime}}$ via $U_{Y^{\prime}} \rightarrow U_{X}$ is trivial.

Proof. Note first that since dilations may be taken to have arbitrarily large tame ramification over the generic points of $D_{X}$, we may assume that $V \rightarrow U_{X}$ is étale over the generic points of $D_{X}$. The rest of the proof will be by induction on $\operatorname{dim}(X)$. (Note that any strictly henselian $X$ is necessarily finite-dimensional.) The case $\operatorname{dim}(X)=1$ is trivial. Now suppose that $\operatorname{dim}(X)=2$. Then the lemma follows from Corollary 1.8 (of $\S 1$ of the present paper), together with the classical purity theorem (i.e., [18], Exposé X, p. 118, Théorème 3.4). Now let us assume that $\operatorname{dim}(X) \geq 3$. We may thus assume that Lemma 3.1 holds for strictly henselian regular $\log$ schemes of dimension $\leq \operatorname{dim}(X)-1$. In particular, it follows that by replacing $(X, M)$ by a dilate of $(X, M)$ (cf. Proposition 1.6 of $\S 1$ ) and applying étale descent, we may assume that $V \rightarrow U_{X}$ extends to a finite étale covering over $X-\{x\}$. Moreover, by completing $(X, M)$ at $x$ (and using faithful flat descent), we may assume that $X$ is the spectrum of a complete, local, strictly henselian ring.

Let $(Y, N)$ be as in Theorem 2.1. Let $\left(X^{*}, M^{*}\right) \rightarrow(X, M)$ be a dilation of $(X, M)$ of magnitude divisible by some positive integer $n$, to be specified below. Let $\left(Y^{*}, N^{*}\right) \rightarrow$ $\left(X^{*}, M^{*}\right)$ be the blow-up of $\left(X^{*}, M^{*}\right)$ (as in Theorem 2.1) constructed using the same nonsingular subdivision $\Delta$ as that used to construct $(Y, N)$ out of $(X, M)$. Thus, we have a commutative diagram:

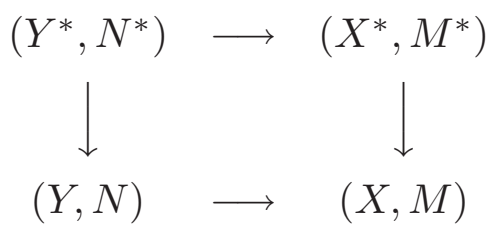

Now I claim that for some appropriate choice of $n$, the morphism $V \rightarrow U_{X}$ extends to a finite étale covering over all the generic points $\mathfrak{p}_{Y^{*}} \in Y^{*}$ of $D_{Y^{*}}$. Indeed, write $\mathfrak{p}_{Y} \in Y$ for the image in $Y$ of $\mathfrak{p}_{Y^{*}}$. If $\mathfrak{p}_{Y}$ maps to a point of $X$ other than $x$, then the claim follows from the fact that $V \rightarrow U_{X}$ already extends over $X-\{x\}$. Thus, we may assume that $\mathfrak{p}_{Y}$ maps to $x \in X$. In particular, we are in the situation of Proposition 2.2. Let $\left(Z^{\prime}, Q^{\prime}\right), \mathfrak{p}_{Z^{\prime}} \in Z^{\prime}$ be as in Proposition 2.2. Now observe that ht $Z_{Z^{\prime}}\left(\mathfrak{p}_{Z^{\prime}}\right)=h_{X}(x)-1=\operatorname{dim}(X)-1$, and that 
$V \rightarrow U_{X}$ extends to a finite étale covering over all of the points of $Z^{\prime}$ of height 1 (since such points map to points of $X$ of height $\leq 2$, by Proposition 2.2, (3), hence to points in $X-\{x\}$ ). Thus, it follows by applying the induction hypothesis (plus étale descent, and the statement at the end of Proposition 2.2) to $Z_{\overline{\mathfrak{p}}_{Z^{\prime}}}^{\prime}$ that for some appropriate choice of $n$, the morphism $V \rightarrow U_{X}$ extends to a finite étale covering over the normalization $Z^{*}$ of $Z_{\mathfrak{p}_{Z^{\prime}}}^{\prime}$ (i.e., the Zariski localization of $Z^{\prime}$ at $\mathfrak{p}_{Z^{\prime}}$ ) in $U_{X^{*}}$. Moreover, since $Y_{\mathfrak{p}_{Y^{*}}}^{*}$ is normal and maps to $Z_{\mathfrak{p}_{Z^{\prime}}}^{\prime}$, it follows that $Y_{\mathfrak{p}_{Y^{*}}}^{*}$ maps to $Z^{*}$. Thus, $V \rightarrow U_{X}$ extends to a finite étale covering over $Y_{\mathfrak{p}_{Y^{*}}}^{*}$, as desired. Since $D_{Y}$ has only finitely many generic points $\mathfrak{p}_{Y}$, taking the product of the various $n$ 's that occur for each $\mathfrak{p}_{Y}$ gives us an " $n$ " for which the claim stated above is valid. This completes the proof of the claim.

To simplify notation, let us replace $(X, M)$ (respectively, $(Y, N))$ by $\left(X^{*}, M^{*}\right)$ (respectively, $\left.\left(Y^{*}, N^{*}\right)\right)$. Thus, we may assume that the morphism $V \rightarrow U_{X}$ extends to a finite étale covering over all the generic points of $D_{Y}$. Since $D_{Y}$ is a divisor with normal crossings in the regular scheme $Y$, we may thus apply the classical purity theorem to conclude that $V \rightarrow U_{X}$ extends to a finite étale covering of $Y$. On the other hand, by Theorem 2.1, $\pi_{1}(Y)$ is trivial. That is to say, $V \rightarrow U_{X}$ extends to a trivial finite étale covering of $Y$. Since $Y$ is birational to $U_{X}$, it thus follows that the covering $V \rightarrow U_{X}$ is trivial, as desired.

Lemma 3.2. In the situation of Lemma 3.1, one may take the integer $n$ to be prime to char $(k)$, the characteristic of the residue field $k$ of $X$ at $x$.

Proof. If $\operatorname{char}(k)=0$, then there is nothing to prove, so let us assume that $\operatorname{char}(k)>$ 0 . Let $p \stackrel{\text { def }}{=} \operatorname{char}(k)$. Fix a clean chart for $(X, M)$, and let $(Y, N) \rightarrow(X, M)$ be a dilation of magnitude $p$. Assume that the covering $V \rightarrow U_{X}$ splits over $Y$. Then it suffices to prove that in this situation, $V \rightarrow U_{X}$ necessarily splits over $X$. Note that there always exists a point $\mathfrak{p} \in X$ of height one such that $p \in \mathfrak{p} \subseteq \Gamma\left(X, \mathcal{O}_{X}\right)$. Let $s_{1}, \ldots, s_{r} \in \Gamma\left(U_{X}, \mathcal{O}_{X}\right)$ be the images of a basis $e_{1} \ldots, e_{r} \in P^{g p}$ (under the composite of $\exp _{M}$ with the clean chart $\left.P^{\mathrm{gp}} \rightarrow M^{\mathrm{gp}}\right)$. Thus, the function field $k(Y)$ of $Y$ is obtained from the function field $k(X)$ of $X$ by adjoining the elements $s_{1}^{\frac{1}{p}}, \ldots, s_{r}^{\frac{1}{p}}$ to $k(X)$. Moreover, $Y$ is the normalization of $X$ in $k(Y)$. For $i=1, \ldots, r$, let $P_{i}$ be the saturation of $P$ in $<P^{\mathrm{gp}}, \frac{1}{p} \cdot e_{1}, \ldots, \frac{1}{p} \cdot e_{i}>\subseteq \frac{1}{p} \cdot P^{\mathrm{gp}}$. Thus, $P_{i}$ is a finitely generated, saturated, integral monoid such that $P_{i}^{\mathrm{gp}}=<P^{\mathrm{gp}}, \frac{1}{p} \cdot e_{1}, \ldots, \frac{1}{p} \cdot e_{i}>$, and $P \subseteq P_{i} \subseteq \frac{1}{p} \cdot P$. Let $\left(Y_{i}, N_{i}\right)$ be the $\log$ scheme obtained from $X$ by letting $Y_{i} \stackrel{\text { def }}{=} X \otimes_{\mathbf{Z}[P]} \mathbf{Z}\left[P_{i}\right]$, and taking the log structure $N_{i}$ to be that associated to the natural chart $P_{i} \rightarrow \mathcal{O}_{Y_{i}}$. One shows as in Lemma 1.4 that $\left(Y_{i}, N_{i}\right)$ is log regular. Thus, we have a chain of morphisms of regular log schemes factorizing $(Y, N) \rightarrow(X, M)$ :

$$
(Y, N)=\left(Y_{r}, N_{r}\right) \rightarrow\left(Y_{r-1}, N_{r-1}\right) \rightarrow \ldots \rightarrow\left(Y_{1}, N_{1}\right) \rightarrow(X, M)
$$

such that each arrow in the chain is finite (over $X$ ) and flat of degree $p$ (over $U_{X}$ ). Note that all the $Y_{i}$ 's are local, normal, and strictly henselian. Thus, since $V \rightarrow U_{X}$ is tamely 
ramified over the generic points of $D_{X}$, it follows that the problem is as follows: Without loss of generality, we may assume that $V \rightarrow U_{X}$ is connected and Galois. Then there is a minimal field $K_{M}$ between the function fields $k(X), k(Y)$ of $X$ and $Y$ such that $V \rightarrow U_{X}$ splits over $K_{M}$. Moreover, $K_{M}$ is unramified over $\mathfrak{p}$. Then we would like to show that $K_{M}=k(X)$.

Thus, by localizing at $\mathfrak{p}$ and considering each of the morphisms $\left(Y_{i+1}, N_{i+1}\right) \rightarrow$ $\left(Y_{i}, N_{i}\right)$, we see that we are reduced to proving the following assertion:

(*) Let $A$ be a discrete valuation ring with residue characteristic $p$. Let $K$ be the quotient field of $A$. Let $L$ be an extension of $K$ of degree $p$ such that the normalization $B$ of $A$ in $L$ is a semilocal Dedekind domain which is obtained from $A$ by adjoining $p^{\text {th }}$ roots of elements of $A$. Then if $K^{\prime} \subseteq L$ is a finite extension of $K$ which is unramified over $A$, then it follows that $K^{\prime}=K$.

Let us prove $\left({ }^{*}\right)$ : First of all, since $[L: K]=p$, if $K^{\prime} \neq K$, it follows that $L=K^{\prime}$. But this implies that $B$ is unramified over $A$. Since $B$ is generated over $A$ by $p^{\text {th }}$ roots of elements of $A$, it follows that the resulting residue field extensions are inseparable. But since they are also separable (as $B$ is étale over $A$ ), it thus follows that they are trivial. Let $\widehat{A}$ (respectively, $\widehat{B}$ ) be the completion of $A$ (respectively, $B$ ). Thus, $\widehat{B}$ is a direct product of $p$ copies of $\widehat{A}$. Relative to this description of $\widehat{B}$, let $b=\left(a_{1}, \ldots, a_{p}\right) \in \widehat{B}$ (where $\left.a_{1}, \ldots, a_{p} \in \widehat{A}\right)$ be such that $b^{p} \in \widehat{A}$. Then it follows that for all $i, j=1, \ldots, p$, we have $a_{i}^{p}=a_{j}^{p}$. Thus, $a_{1}, \ldots, a_{p}$ all have the same image in the residue field of $\widehat{A}$. On the other hand, since $\widehat{B}$ is generated over $\widehat{A}$ by such $b$, it follows that any element $\left(c_{1}, \ldots, c_{p}\right) \in \widehat{B}$ (where $c_{1}, \ldots, c_{p} \in \widehat{A}$ ) satisfies the condition that the $c_{i}$ all have the same image in the residue field of $\widehat{A}$. But this is absurd. This completes the proof of $(*)$, and hence of the lemma.

The following is an unpublished result of K. Kato:

Theorem 3.3. (Log Purity Theorem) Let $(X, M)$ be a regular log scheme. Let $U_{Y} \rightarrow U_{X}$ be a finite étale covering of the interior $U_{X}$ of $X$ which is tamely ramified over the generic points of $D_{X} \rightarrow X$. Let $Y$ be the normalization of $X$ in $U_{Y}$. Then the pair $\left(Y, U_{Y}\right)$ is toric (cf. Definition 1.2), hence defines a regular log scheme $(Y, N)$. Moreover, $U_{Y} \rightarrow U_{X}$ extends uniquely to a log étale morphism $(Y, N) \rightarrow(X, M)$.

Proof. Clearly, one may assume that $X$ is strictly henselian. Thus, by Lemmas 3.1 and 3.2 , it follows that there exists an integer $n$ which is invertible on $X$ such that for some dilation $(Z, L) \rightarrow(X, M)$ of magnitude $n, U_{Y} \rightarrow U_{X}$ splits over $U_{Z}$. Let $P^{\mathrm{gp}} \rightarrow M^{\mathrm{gp}}$ be the clean chart (cf. Definition 1.3) used to define this dilation. Let us assume (without loss of generality) that $U_{Y}$ is connected. Then the function field $k(Y)$ of $U_{Y}$ lies between the function fields $k(X)$ (of $X$ ) and $k(Z)$ (of $Z$ ). Moreover, since the extension $k(Z) / k(X)$ 
is abelian with Galois group equal to $\left(\frac{1}{n} \cdot P^{\mathrm{gp}}\right) / P^{\mathrm{gp}}$ (tensored with the group of $n^{\text {th }}$ roots of unity), it follows that the intermediate field $k(Y)$ corresponds to some submodule $P_{Y}^{\mathrm{gp}}$ of $\frac{1}{n} \cdot P^{\text {gp }}$ that contains $P^{\text {gp }}$. Let us write $P_{Y}$ for the smallest saturated submonoid of $P_{Y}^{\mathrm{gp}}$ containing $P$. Thus, we have $P \subseteq P_{Y} \subseteq \frac{1}{n} \cdot P ; P_{Y}^{\mathrm{gp}}$ is the group associated to $P_{Y}$; and $P_{Y}$ is a finitely generated integral, saturated monoid. Moreover, if we equip $Y^{\prime} \stackrel{\text { def }}{=} X \times_{\mathbf{Z}[P]} \mathbf{Z}\left[P_{Y}\right]$ with the log structure $N^{\prime}$ associated to the natural chart defined by $P_{Y}$, one sees immediately (cf. the proof of Lemma 1.4) that $\left(Y^{\prime}, N^{\prime}\right)$ is log regular, and that the natural morphism $\left(Y^{\prime}, N^{\prime}\right) \rightarrow(X, M)$ is log étale. Moreover, since $U_{Y^{\prime}}$ may be identified with $U_{Y}$, and $Y^{\prime}$ is necessarily normal, it follows that $Y^{\prime}$ may be identified with $Y$, and that $\left(Y, U_{Y}\right)=\left(Y^{\prime}, U_{Y^{\prime}}\right)$ is toric. This completes the proof of Theorem 3.3.

\section{$\S 4$. Families of Stable Curves}

Let $S$ be a noetherian scheme. Let $f: X \rightarrow S$ be a morphism of finite type, and $D_{M} \subseteq X$ a closed subscheme.

Definition 4.1. We shall call $\left(f: X \rightarrow S, D_{M}\right)$ locally stable if

(1) $f$ is flat;

(2) the geometric fibers of $f$ are reduced, connected, one-dimensional, and have at most nodes as singularities;

(3) $f$ is smooth at the points of $D_{M}$, and $D_{M}$ is a union of the images of disjoint sections $s_{1}, \ldots, s_{r}: S \rightarrow X$.

We shall call $D_{M}$ the divisor of marked points. When $D_{M}=\emptyset$, we shall simply say that $f$ is locally stable.

Note that if $\left(f, D_{M}\right)$ is locally stable, then $f$ is locally stable. It is easy to check that if $f$ is locally stable, then it is a local complete intersection, and in particular, Gorenstein. Thus, its dualizing sheaf ([12]) forms a natural line bundle $\omega_{X / S}$ on $X$. In general, if $\left(f, D_{M}\right)$ is locally stable, then $D_{M} \subseteq X$ forms an $S$-flat divisor in $X$; thus, one may consider the line bundle $\omega_{X / S}\left(D_{M}\right)$ on $X$.

We remark that the local structure of locally stable morphisms is well-known (cf. [3]). Indeed, suppose that $S=\operatorname{Spec}(A)$, where $A$ is a complete local ring with separably closed residue field. Suppose further that $\left(f: X \rightarrow S, D_{M}\right)$ is locally stable, and that $x \in X$ is a closed point of $X$ lying over the closed point $s \in S$ of $S$, and write $B \stackrel{\text { def }}{=} \widehat{\mathcal{O}}_{X, x}$ (the completed local ring of $X$ at $x$ ). Then there are three possibilities for the local structure of $\left(X \rightarrow S, D_{M}\right)$ : 
(1) $f$ is smooth at $x$ and $x \notin D_{M}$ : Then $B$ is $A$-isomorphic to $A[[t]]$ (where $t$ is an indeterminate).

(2) $f$ is smooth at $x$ and $x \in D_{M}$ : Then $B$ is $A$-isomorphic to $A[[t]]$ (where $t$ is an indeterminate) and $D_{M}=V(t)$ (i.e., $D_{M}$ is the closed subscheme defined by $t$ ).

(3) $f$ is not smooth at $x$ : Then $B$ is $A$-isomorphic to $A[[s, t]] /(s t-\alpha)$, where $s$ and $t$ are indeterminates and $\alpha \in \mathfrak{m}_{A}$ (the maximal ideal of $A$ ).

In fact, for an arbitrary locally stable $\left(f: X \rightarrow S, D_{M}\right)$, functions such as " $s$ " and " $t$ " can be found after étale localization on $X$ and $S$, i.e., one need not go all the way to the completions of $X$ and $S$ at the points in question.

Lemma 4.2. Suppose that $\left(S, U_{S}\right)$ is a toric pair (Definition 1.2) - i.e., $U_{S}$ is the interior of $S$. Let $\left(f: X \rightarrow S, D_{M}\right)$ be locally stable, such that $f$ is smooth over $U_{S} \subseteq S$. Then if $U_{X} \stackrel{\text { def }}{=} f^{-1}\left(U_{S}\right) \bigcap\left(X-D_{M}\right) \subseteq X$ (thus, in general, $\left.U_{X} \neq X-D_{M}\right)$, then $\left(X, U_{X}\right)$ is a toric pair. In particular, $X$ is normal.

Proof. We begin by proving that $X$ is normal. First, we observe that (by the above discussion of the local structure of $f$ ) $f$ is a local complete intersection morphism. Thus, to check that $X$ is normal, it suffices to check that $X$ is regular at all of its height one primes (i.e., points). Since $f$ is flat, such primes map to primes of height $\leq 1$ of $S$. Since $S$ is normal, such primes of $S$ will be regular. Moreover, since $f$ is smooth over $U_{S}$, hence over all generic points of $S$, it follows (by the above discussion of the local structure of $f$ ) that it suffices to check the following: If $A$ is a field or a complete discrete valuation ring, then $A[[t]]$ and $A[[s, t]] /(s \cdot t-\alpha)$ (where $s$ and $t$ are indeterminates and $0 \neq \alpha \in \mathfrak{m}_{A}$ ) are regular at primes of height one. But this is clear. This completes the proof that $X$ is normal.

Now let us consider the monoid

$$
M \stackrel{\text { def }}{=} \mathcal{O}_{X} \bigcap i_{*} \mathcal{O}_{U_{X}}^{\times} \subseteq i_{*} \mathcal{O}_{U_{X}}
$$

(where $i: U_{X} \hookrightarrow X$ is the natural inclusion). It follows easily from the above discussion of the local structure of $f$ that $M$ defines a fine log structure on $X$. Indeed, in case (1) above, this $\log$ structure is simply the pull-back to $X$ of the $\log$ structure on $S$. In case (2) above, this log structure is the result of adjoining (to the pull-back to $X$ of the $\log$ structure on $S$ ) a single copy of $\mathbf{N}$, corresponding to some defining equation (i.e., " $t$ " in the above discussion) of the divisor $D_{M}$. In case (3) above, this log structure is the result of adjoining (to the pull-back to $X$ of the $\log$ structure on $S$ ) two copies of $\mathbf{N}$, corresponding (in the notation of the above discussion) to " $s$ " and " $t$," together with a single relation, corresponding to the equation "st $-\alpha$." Let us denote the resulting log scheme by $X^{\log }$. 
Since $X$ is normal, it follows immediately (from the above definition of the monoid $M$ ) that $M$ is saturated.

Next, let us observe that we have a log morphism

$$
X^{\log } \rightarrow S^{\log }
$$

(of saturated fine log schemes). Moreover, it follows easily from the local descriptions of the preceding paragraph that this morphism is log smooth. Thus, we conclude (by [Kato2], Theorem 8.2) that $X^{\log }$ is $\log$ regular, as desired.

Definition 4.3. We shall say that $\left(f, D_{M}\right)$ is stable or a stable curve if $\left(f, D_{M}\right)$ is locally stable, $f$ is proper, and $\omega_{X / S}\left(D_{M}\right)$ is relatively ample over $S$.

Suppose that $\left(f, D_{M}\right)$ is stable. Then note that $f_{*} \omega_{X / S}$ forms a vector bundle on $S$. Naturally, the rank of this vector bundle is locally constant. When it is constant, we shall refer to this rank as the genus $g$ of $\left(f, D_{M}\right)$. The degree of the divisor $D_{M}$ over $S$ is also locally constant on $S$. When it is constant, we shall refer to this degree as the number of marked points $r$ of $\left(f, D_{M}\right)$. One checks easily that the above definition of a stable curve is equivalent to that given in [3] (when $D_{M}=\emptyset$ ) or, more generally, a pointed stable curve, as in [10] (except that in the above definition we did not fix the genus or the number of marked points, and we did not assume that we were given an ordering of the marked points). In particular, as one knows from [3] and [10], there is a natural moduli stack

$$
\overline{\mathcal{M}}_{g, r} \rightarrow \operatorname{Spec}(\mathbf{Z})
$$

of $r$-pointed stable curves of genus $g$ over $\mathbf{Z}$. Moreover, it follows from the condition that $\omega_{X / S}(D)$ be relatively ample that

$$
2 g-2+r \geq 1
$$

Now let $U$ be an $S$-scheme of finite type.

Definition 4.4. We shall call $U \rightarrow S$ a hyperbolic curve if there exists a surjective étale morphism $T \rightarrow S$, together with a stable curve $(Y \rightarrow T, D)$ such that: (i) $Y \rightarrow T$ is smooth; and (ii) $U_{T} \stackrel{\text { def }}{=} U \times_{S} T$ is $T$-isomorphic to $Y-D$.

In this paper, the prefix "poly-" will be used to mean "successive extensions or families of." Thus, we have the following generalizations of "stable curves" and "hyperbolic curves": Let $f: X \rightarrow S$ be a morphism of finite type, and $D \subseteq X$ a closed subscheme. 
Definition 4.5. We shall say that $(f: X \rightarrow S, D)$ is polystable or a stable polycurve if the following data exist: a factorization

$$
X=X_{0} \quad \stackrel{f_{0}}{\longrightarrow} X_{1} \stackrel{f_{1}}{\longrightarrow} X_{2} \quad \ldots \quad X_{i} \stackrel{f_{i}}{\longrightarrow} \quad X_{i+1} \quad \ldots \quad X_{n} \stackrel{f_{n}}{\longrightarrow} \quad X_{n+1}=S
$$

together with a decomposition

$$
D=\bigcup_{i=0}^{n+1} \phi_{i}^{-1}\left(D_{i}\right)
$$

(where $D_{i} \subseteq X_{i}$ is a closed subscheme; $\phi_{0}: X \rightarrow X$ is the identity; and, for each $i=$ $1, \ldots, n+1$, we let $\phi_{i} \stackrel{\text { def }}{=} f_{i-1} \circ f_{i-2} \circ \ldots \circ f_{1} \circ f_{0}: X=X_{0} \rightarrow X_{i}$ ) of $D$ into a (schematic) union of closed subschemes, satisfying the following properties:

(1) For each $i=0, \ldots, n$, the pair $\left(f_{i}: X_{i} \rightarrow X_{i+1}, D_{i}\right)$ is a stable curve. Moreover, $S$ is normal in a neighborhood of $D_{n+1}$, and $D_{n+1}$ is a reduced divisor in $\mathrm{S}$.

(2) Let $U_{n+1} \stackrel{\text { def }}{=} X_{n+1}-D_{n+1} \subseteq X_{n+1}=S$. For $i=0, \ldots, n$, if $U_{i+1} \subseteq$ $X_{i+1}$ is defined, then let $U_{i} \stackrel{\text { def }}{=} f_{i+1}^{-1}\left(U_{i+1}\right) \cap\left(X_{i}-D_{i}\right) \subseteq X_{i}$. Then we assume that (for each $i=0, \ldots, n) f_{i}$ is smooth over $U_{i+1}$ (for $i=$ $0, \ldots, n)$.

We shall refer to $U_{X} \stackrel{\text { def }}{=} X-D=U_{0} \subseteq X_{0}=X$ as the interior of $X$.

In particular, it follows from Lemma 4.2 if $\left(S, U_{n+1}\right)$ is a toric pair, and $(f, D)$ is polystable, then $\left(X, U_{0}\right)$ it also a toric pair. In this situation, we shall say " $(f, D)$ is polystable over $\left(S, U_{n+1}\right)$," or that " $\left(X, U_{0}\right) \rightarrow\left(S, U_{n+1}\right)$ is polystable." If $\left(X, M_{X}\right)$ and $\left(S, M_{S}\right)$ are the $\log$ schemes associated to $\left(X, U_{0}\right)$ and $\left(S, U_{n+1}\right)$, respectively, then we shall say that " $\left(X, M_{X}\right) \rightarrow\left(S, M_{S}\right)$ is (log) polystable."

Now let $U$ be an $S$-scheme of finite type.

Definition 4.6. We shall say that $f: U \rightarrow S$ is polyhyperbolic or a hyperbolic polycurve if the following data exist: a factorization

$$
U=U_{0} \stackrel{f_{0}}{\longrightarrow} U_{1} \stackrel{f_{1}}{\longrightarrow} U_{2} \quad \ldots \quad U_{i} \stackrel{f_{i}}{\longrightarrow} U_{i+1} \quad \ldots \quad U_{n} \stackrel{f_{n}}{\longrightarrow} U_{n+1}=S
$$


such that each $f_{i}$ (for $\left.i=0, \ldots, n\right)$ is a hyperbolic curve.

Remark. $\quad$ Some people are in the habit of referring to hyperbolic polycurves as "Artin neighborhoods." In fact, strictly speaking, however, these two notions are not equivalent: Indeed, in the definition of an Artin neighborhood, the morphisms $f_{i}$ (of Definition 4.6) are required to be affine. On the other hand, in the definition of an Artin neighborhood, the genus $g$ and number of marked points $r$ need not satisfy the inequality $2 g-2+r \geq 1$. Thus, neither of these two notions is stronger than the other.

\section{$\S 5$. Functoriality of the Locus of Indeterminacy}

Let $A$ be a noetherian ring. Let $Z$ be an integral, projective $A$-scheme of finite type. Thus, we assume that $Z$ admits a closed immersion $Z \hookrightarrow \mathbf{P}_{A}^{N}$ into some projective space over $A$. In particular, there exists a very ample line bundle $\mathcal{L}$ on $Z$.

Let $X$ and $Y$ be normal, connected, separated noetherian schemes. Let

$$
\alpha: Y \rightarrow X
$$

be a finite morphism. Suppose that we are given also given a morphism

$$
f_{U}: U \rightarrow Z
$$

defined on some open subscheme $U \subseteq X$ such that $X-U$ has codimension $\geq 2$ in $X$. Since $Z$ is separated, it follows that any extension of $f_{U}$ to an open subset $U^{\prime} \subseteq X$ that contains $U$ is unique. Thus, there exists a maximal open subscheme $\bar{U} \subseteq X$ to which $f_{U}$ extends.

Definition 5.1. We shall call $\bar{U}$ the locus of determinacy for $f_{U}$, and we shall call its complement $X-\bar{U}$ (a closed subset of $X$ ) the locus of indeterminacy for $f_{U}$.

Let $V \stackrel{\text { def }}{=} \alpha^{-1}(U) \subseteq Y$. Let us suppose that $Y-V$ has codimension $\geq 2$ in $Y$. Thus, we can define $\bar{V} \subseteq Y$, i.e., the locus of determinacy for $\alpha \circ f_{U}$. Then it is natural to ask what the relationship is between $\bar{U}$ and $\bar{V}$. On the one hand, it follows tautologically from the definitions that

$$
f^{-1}(\bar{U}) \subseteq \bar{V}
$$

The reverse inclusion is more subtle. In order to analyze the reverse inclusion, let us consider the line bundle $\mathcal{L}_{U} \stackrel{\text { def }}{=} f_{U}^{*} \mathcal{L}$. Note that since $X$ is normal and $X-U$ has codimension $\geq 2$ in $X$, it follows that any extension of $\mathcal{L}_{U}$ to an open subset of $X$ containing 
$U$ is unique. Note further that it is clear (from the fact that $f_{U}$ extends to a morphism $f_{\bar{U}}: \bar{U} \rightarrow Z$ ) that $\mathcal{L}_{U}$ extends to a line bundle $\mathcal{L}_{\bar{U}}$ on $\bar{U}$.

Lemma 5.2. $\quad$ Suppose that $\mathcal{L}_{\bar{U}}$ extends to a line bundle $\mathcal{L}_{X}$ on $X$. Then it follows that $\bar{V}=\alpha^{-1}(\bar{U})$.

Proof. Note that since $Z$ is projective over $A$, it follows that $\Gamma(Z, \mathcal{L})$ is a finitely generated $A$-module. Let $s_{1}, \ldots, s_{N} \in \Gamma(Z, \mathcal{L})$ be a set of generating sections of $\Gamma(Z, \mathcal{L})$. Note that each $s_{i}$ may be pulled back to a section $\left.s_{i}\right|_{U} \in \Gamma\left(U, \mathcal{L}_{U}\right)$ which extends uniquely (since $X$ is normal) to a section $\left.s_{i}\right|_{X} \in \Gamma\left(X, \mathcal{L}_{X}\right)$. Now I claim that:

(*) The closed subset $F_{X} \stackrel{\text { def }}{=} X-\bar{U} \subseteq X$ coincides with the common zero locus $N_{X}$ (regarded as a closed subset of $X$ ) of the sections $\left.s_{1}\right|_{X}, \ldots,\left.s_{N}\right|_{X}$ of $\mathcal{L}_{X}$ over $X$.

Indeed, since $s_{1}, \ldots, s_{N}$ have no common zeroes on $Z$, one concludes immediately that $\left.s_{1}\right|_{X}, \ldots,\left.s_{N}\right|_{X}$ have no common zeroes on $\bar{U}$, so $N_{X} \subseteq F_{X}$. On the other hand, over the open subscheme $U^{\prime} \stackrel{\text { def }}{=} X-N_{X} \subseteq X$, since $\left.s_{1}\right|_{U^{\prime}}, \ldots,\left.s_{N}\right|_{U^{\prime}}$ have no common zeroes on $U^{\prime}$, they define a morphism $U^{\prime} \rightarrow \mathbf{P}_{A}^{N}$ whose image is contained in $Z \hookrightarrow \mathbf{P}_{A}^{N}$ (where the inclusion " $\hookrightarrow$ " is that defined by $\left.s_{1}, \ldots, s_{N}\right)$ and whose restriction to $U$ is $f_{U}$. Thus, $U^{\prime} \subseteq \bar{U}$, so $F_{X} \subseteq N_{X}$. Thus, $F_{X}=N_{X}$. This completes the proof of the claim.

Now let $F_{Y} \stackrel{\text { def }}{=} Y-\bar{V} \subseteq Y$ (a closed subset of $Y$ ). Note that $\mathcal{L}_{\bar{V}}$ extends (uniquely) to a line bundle $\mathcal{L}_{Y}=\alpha^{*} \mathcal{L}_{X}$ on $Y$. Thus, we have sections $\left.s_{1}\right|_{Y}, \ldots,\left.s_{N}\right|_{Y} \in \Gamma\left(Y, \mathcal{L}_{Y}\right)$. Let $N_{Y}$ be the common zero locus $N_{Y}$ (regarded as a closed subset of $Y$ ) of the sections $\left.s_{1}\right|_{Y}, \ldots,\left.s_{N}\right|_{Y}$ of $\mathcal{L}_{Y}$ over $Y$. Then by the same argument as that given for $\left(^{*}\right)$ above, we have that $F_{Y}=N_{Y}$. On the other hand, since $\left.s_{i}\right|_{Y}=\alpha^{*}\left(\left.s_{i}\right|_{X}\right)$ (for $\left.i=1, \ldots, N\right)$, it is clear that $N_{Y}=\alpha^{-1}\left(N_{X}\right)$ (as closed subsets). Thus, we obtain that $F_{Y}=\alpha^{-1}\left(F_{X}\right)$, hence that $\bar{V}=\alpha^{-1}(\bar{U})$, as desired.

Corollary 5.3. Suppose that $\alpha: Y \rightarrow X$ is dominant, and $\bar{V}=Y$. Then $\bar{U}=X$.

Proof. Since $\alpha$ is finite and dominant, and $X$ is integral, it follows that $\alpha$ is surjective. Since $f_{U}$ extends to a morphism $Y \rightarrow Z$, it follows that $\mathcal{L}_{\bar{V}}$ extends to a line bundle $\mathcal{L}_{Y}$ on $Y$. Thus, by taking the norm of $\mathcal{L}_{Y}$, we obtain a line bundle on $X$ which extends some tensor power of $\mathcal{L}_{\bar{U}}$. In particular, by replacing the original very ample $\mathcal{L}$ by a power of $\mathcal{L}$, we see that we can apply Lemma 5.2. Thus, $\bar{U}=\alpha\left(\alpha^{-1}(\bar{U})\right)=\alpha(\bar{V})=\alpha(Y)=X$, as desired.

In fact, we shall often wish to apply Lemma 5.2 (or Corollary 5.3) when $Z$ is not necessarily a projective scheme over $A$, but instead an integral algebraic stack over $A$ whose associated coarse moduli space (as in [4], Chapter 1, Theorem 4.10) is a projective 
scheme over $A$. In fact, the main example that we have in mind is the moduli stack $\overline{\mathcal{M}} \stackrel{\text { def }}{=} \overline{\mathcal{M}}_{g, r} \rightarrow \operatorname{Spec}(\mathbf{Z})$ (for some nonnegative integers $g$ and $r$ such that $2 g-2+r \geq 1$ - cf. $\S 1$ ), whose associated coarse moduli space $\overline{\mathcal{M}}^{\mathrm{c}}$ is well-known to be projective over Z (cf. [16], Corollary 5.18). Unfortunately, we cannot apply Lemma 5.2 or Corollary 5.3 immediately in the case of a general algebraic stack $Z$, but in the special case of $\overline{\mathcal{M}}$, we can still make use of Lemma 5.2 or Corollary 5.3 by applying Lemma 5.2 /Corollary 5.3 in tandem with Lemma 5.4 or Lemma 5.5 below.

Let $l \geq 3$ be a prime number. Let $\mathcal{M} \subseteq \overline{\mathcal{M}}$ be the open substack parametrizing smooth curves. Let $\mathcal{C} \rightarrow \mathcal{M}$ be the tautological curve over $\mathcal{M}$. Let $\mathcal{J} \rightarrow \mathcal{M}$ be the Jacobian of the tautological curve. Write $\mathcal{J}[l]$ for the kernel of the map $\mathcal{J} \rightarrow \mathcal{J}$ given by multiplication by $l$. Thus, $\mathcal{J}[l] \otimes \mathbf{Z}\left[\frac{1}{l}\right]$ is étale over $\mathcal{M} \otimes \mathbf{Z}\left[\frac{1}{l}\right]$, and may be thought of as a local system of abelian groups isomorphic to $(\mathbf{Z} / l \cdot \mathbf{Z})^{2 g}$. Write $\mathcal{M}[l] \rightarrow \mathcal{M} \otimes \mathbf{Z}\left[\frac{1}{l}\right]$ for the finite étale covering of "level structures," i.e., isomorphisms of this local system with $(\mathbf{Z} / l \cdot \mathbf{Z})^{2 g}$. Thus, $\mathcal{M}[l]$ is étale over $\mathcal{M} \otimes \mathbf{Z}\left[\frac{1}{l}\right]$, and tamely ramified over the divisor at infinity of $\overline{\mathcal{M}} \otimes \mathbf{Z}\left[\frac{1}{l}\right]$ (cf., e.g., $§ 3.22,3.23$, of $[14]$ ).

The following two lemmas are well-known (cf., e.g., Lemma 2.1 of [1]):

Lemma 5.4. $\quad$ Let $X$ be a normal, connected, separated noetherian scheme with generic point $\eta$. Let $f_{\eta}: \eta \rightarrow \overline{\mathcal{M}}$ be a morphism that lifts to a morphism $h_{\eta}: \eta \rightarrow \mathcal{M}[l]$, where $l$ is invertible on $X$. Write $f_{\eta}^{\mathrm{c}}: \eta \rightarrow \overline{\mathcal{M}}^{\mathrm{c}}$ for the composite of $f_{\eta}$ with $\overline{\mathcal{M}} \rightarrow \overline{\mathcal{M}}^{\mathrm{c}}$. Suppose further that $f_{\eta}^{\mathrm{c}}$ extends to a morphism $f^{\mathrm{c}}: X \rightarrow \overline{\mathcal{M}}^{\mathrm{c}}$. Then $f_{\eta}$ extends uniquely to a morphism $f: X \rightarrow \overline{\mathcal{M}}$.

Proof. Note that since $\overline{\mathcal{M}}=\overline{\mathcal{M}}^{\mathrm{c}}$ when $g=0$, we may assume that $g \geq 1$. Moreover, since $\overline{\mathcal{M}}_{g, r} \rightarrow \overline{\mathcal{M}}_{g, r-1}$ (where $2 g-2+r-1 \geq 1$ ) is projective, it follows that we may assume that either $r=0$, or $g=r=1$. Thus, in the following we assume that either $r=0$ or $g=r=1$.

By standard techniques (e.g., replacing $X$ by a scheme of finite type over $\mathbf{Z}$, completing at a prime, and then enlarging the residue field), we may assume that $X=\operatorname{Spec}(R)$, where $R$ is a complete local ring with separably closed residue field. Let us denote the closed point of $X$ by $x$, and the quotient ring of $R$ by $K$. Let $M^{\mathrm{c}}$ be the strict henselization of $\overline{\mathcal{M}}^{\mathrm{c}}$ at $f^{\mathrm{c}}(x)$; let $M \stackrel{\text { def }}{=} \overline{\mathcal{M}} \times \overline{\mathcal{M}}^{\mathrm{c}} M^{\mathrm{c}}$. Thus, there is a strict henselian regular local ring $Q$, equipped with the action of a finite group $G$, such that $M$ is the quotient of $M^{\prime} \stackrel{\text { def }}{=} \operatorname{Spec}(Q)$ by $G$ "in the sense of stacks," and $M^{\mathrm{c}}=\operatorname{Spec}\left(Q^{G}\right)$ (cf. [4], Chapter 1, Theorem 4.10). Here, $G$ is the automorphism group of the curve $f^{\mathrm{c}}(x)$ (cf. [4], Chapter 1, Theorem 4.10). Moreover, $f^{\mathrm{c}}$ factors through $M^{\mathrm{c}}$. Thus, since $M=\overline{\mathcal{M}} \times \overline{\mathcal{M}}^{\mathrm{c}} M^{\mathrm{c}}$, it follows that $f_{\eta}$ factors through $M$. Denote the resulting morphism by $f_{\eta}^{M}: \eta \rightarrow M$. Now I claim that:

(*) The morphism $f_{\eta}^{M}: \eta \rightarrow M$ factors through $M^{\prime}=\operatorname{Spec}(Q)$. 
Before proving this claim, let us observe that this claim completes the proof of Lemma 5.4. Indeed, the claim states that $f_{\eta}^{M}: \eta \rightarrow M$ factors through some morphism $\eta \rightarrow M^{\prime}$. Moreover, since $Q$ is finite over $Q^{G}, X$ is normal, and we have a morphism $X \rightarrow M^{\mathrm{c}}$, we thus conclude that the morphism $\eta \rightarrow M^{\prime}$ extends to a morphism $X \rightarrow M^{\prime}$. Thus, in particular, $f_{\eta}^{M}: \eta \rightarrow M$ extends to a morphism $X \rightarrow M$, so $f_{\eta}: \eta \rightarrow \overline{\mathcal{M}}$ extends to a morphism $f: X \rightarrow \overline{\mathcal{M}}$, as desired. The uniqueness statement follows from the fact that $\overline{\mathcal{M}}$ is a separated algebraic stack.

Now let us prove (*). Let $C \rightarrow M$ be the tautological curve. Write $m \in M$ for the closed point of $M$, and $C_{m}$ for the fiber of $C \rightarrow M$ over $m$. Note that since $l$ is invertible on $X$, it follows that any line bundle $\mathcal{L}_{m}$ on $C_{m}$ such that $\mathcal{L}_{m}^{\otimes l} \cong \mathcal{O}_{C_{m}}$ lifts uniquely to a line bundle $\mathcal{L}^{\prime}$ on $C^{\prime} \stackrel{\text { def }}{=} C \times_{M} M^{\prime}$ such that $\left(\mathcal{L}^{\prime}\right)^{\otimes l} \cong \mathcal{O}_{C^{\prime}}$. It thus follows that the "line bundles on $C$ whose $l^{\text {th }}$ power is trivial" form an étale local system on $M$ which is étale locally isomorphic to $(\mathbf{Z} / l \cdot \mathbf{Z})^{a}$, for some nonnegative integer $a \leq 2 g$. Let us denote by $N \rightarrow M$ the finite étale covering parametrizing isomorphisms of this local system to $(\mathbf{Z} / l \cdot \mathbf{Z})^{a}$. Thus, $N$ is a stack parametrizing curves equipped with line bundles satisfying certain properties. By [2], Corollaire 5.17, and [3], Theorem 1.13, the curve plus line bundle pairs corresponding to the closed points of $N$ have no automorphisms. Thus, $N$ is in fact a scheme. More precisely, $N$ is a finite disjoint union of spectra of strictly henselian regular local rings. Since $M^{\prime} \rightarrow M$ is finite étale, it thus follows that the morphism $N \rightarrow M$ factors through $M^{\prime}$.

Thus, to prove $\left(^{*}\right)$, it suffices to prove that $f_{\eta}^{M}: \eta \rightarrow M$ factors through $N$. But this is a consequence of the fact that the l-torsion points of the Jacobian of the curve $C_{\eta} \stackrel{\text { def }}{=} C \times_{M} \eta$ are all defined over $\eta$ (by the existence of $h_{\eta}$ ). This completes the proof of $(*)$, and hence of Lemma 5.4.

Lemma 5.5. $\quad$ Let $X$ be an integral separated noetherian scheme with generic point $\eta$. Suppose, moreover, that $X$ has the following property:

(*) The completions of the local rings of $X$ are normal complete intersections.

Let $f_{\eta}: \eta \rightarrow \overline{\mathcal{M}}$ be a morphism. Assume that either

(1) $f_{\eta}$ extends over all the points of $X$ of height $\leq 2$; or

(2) $X$ is regular and $f_{\eta}$ extends over all the points of $X$ of height $\leq 1$.

Write $f_{\eta}^{\mathrm{c}}: \eta \rightarrow \overline{\mathcal{M}}^{\mathrm{c}}$ for the composite of $f_{\eta}$ with $\overline{\mathcal{M}} \rightarrow \overline{\mathcal{M}}^{\mathrm{c}}$. Suppose further that $f_{\eta}^{\mathrm{c}}$ extends to a morphism $f^{\mathrm{c}}: X \rightarrow \overline{\mathcal{M}}^{\mathrm{c}}$. Then $f_{\eta}$ extends uniquely to a morphism $f: X \rightarrow \overline{\mathcal{M}}$.

Proof. By $\left(^{*}\right)$, we reduce immediately (by descent) to the case where $X=\operatorname{Spec}(R)$, where $R$ is a complete local ring with separably closed residue field. Now let us use the 
notation of the proof of Lemma 5.4. Just as in the proof of Lemma 5.4, it suffices to show that the morphism $f_{\eta}^{M}: \eta \rightarrow M$ factors through $M^{\prime}=\operatorname{Spec}(Q)$. Note that $M^{\prime} \rightarrow M$ is a finite étale morphism. If we pull it back via $f_{\eta}^{M}$, we thus get a finite étale morphism $\eta^{\prime} \rightarrow \eta$. Clearly, it suffices to show that $\eta^{\prime} \rightarrow \eta$ admits a section. But note that the assumptions of the lemma imply that $\eta^{\prime} \rightarrow \eta$ extends to a finite étale covering either over the primes of $R$ of height $\leq 2$ (under assumption (1)) or over the primes of $R$ of height $\leq 1$ (under assumption (2), in which case $R$ is regular). In either case, by [18], Exposé $\mathrm{X}, \mathrm{p}$. 118, Théorème 3.4, it follows that $\eta^{\prime} \rightarrow \eta$ extends to a finite étale covering $X^{\prime} \rightarrow X$ over $X=\operatorname{Spec}(R)$. Since $X$ is strictly henselian, this covering thus admits a section. Thus, $\eta^{\prime} \rightarrow \eta$ also admits a section, as desired.

Remark. We would like to apply Lemma 5.5 when $X$ has toric singularities (cf. Definition 1.2). Observe that in this case, (since the completion (at a point) of a regular $\log$ scheme is again a regular log scheme) $X$ automatically satisfies the normality part of the assumption $\left(^{*}\right)$ in Lemma 5.5 (cf. the discussion following Definition 1.1).

Before continuing, we make a definition which will be important in the following $\S$ 's: Let $X$ be a normal noetherian scheme. Let $D \subseteq X$ be an effective Weil divisor in $X$, and let $U \stackrel{\text { def }}{=} X-D$. Thus, $U$ is a dense open subscheme of $X$. Suppose that we are given a morphism

$$
h_{U}: U \rightarrow \overline{\mathcal{M}}
$$

such that $h_{U}(U) \subseteq \mathcal{M}$.

Definition 5.6. Let $l$ be a prime number. We shall say that $h_{U}$ admits a level $l$ structure if it factors through $\mathcal{M}[l]$.

Note that it follows from [2], Corollaire 5.18 and Proposition 5.7 (and the fact that $\overline{\mathcal{M}}_{g, r+1} \rightarrow \overline{\mathcal{M}}_{g, r}$ is projective) that if $h_{U}$ admits a level $l$ structure, then $h_{U}$ extends (as a morphism to $\overline{\mathcal{M}}$ ) over the generic points of $D$.

Finally, we include one more (well-known) result concerning the locus of indeterminacy which we will use in the following. Let $X, U, Z$, and $A$ be as above. Moreover, just as above, we assume that we are given a morphism

$$
f_{U}: U \rightarrow Z
$$

Assume further that $X$ has the structure of an $A$-scheme, and that $f_{U}$ is a morphism of $A$-schemes. Then we have the following result: 
Lemma 5.7. $\quad$ Suppose that $\phi: X^{\prime} \rightarrow X$ is a projective morphism between normal, connected, separated noetherian schemes which is an isomorphism over $U$. Suppose, moreover, that $f_{U}: U \rightarrow Z$ extends to $X^{\prime}$ and that for each $x \in X$, this extension of $f_{U}$ maps $\phi^{-1}(x) \subseteq X^{\prime}$ to a point. Then $f_{U}$ extends to $X$.

Proof. Let $\Gamma \subseteq X \times_{A} Z$ be the closure (with the reduced induced scheme structure) in $X \times{ }_{A} Z$ of the graph $\Gamma_{U} \subseteq U \times{ }_{A} Z$ of $f_{U}$. Let $\Gamma^{\prime} \subseteq X^{\prime} \times_{A} Z$ be the graph of the extension to $X^{\prime}$ of $f_{U}$. Let $U^{\prime} \stackrel{\text { def }}{=} \phi^{-1}(U)$. Thus, $U^{\prime} \cong U$. Since $\Gamma^{\prime}$ is the closure of the graph $\Gamma_{U}^{\prime}$ of the composite of $U^{\prime} \cong U$ with $f_{U}$, and $\Gamma_{U}^{\prime}$ maps isomorphically to $\Gamma_{U}$ under the morphism $\phi_{Z}: X^{\prime} \times_{A} Z \rightarrow X \times_{A} Z$, it follows that $\phi_{Z}$ maps $\Gamma^{\prime}$ into $\Gamma$. Thus, we have a morphism

$$
\beta: X^{\prime} \cong \Gamma^{\prime} \rightarrow \Gamma
$$

together with a projection morphism $\pi: \Gamma \rightarrow X$ such that $\pi \circ \beta=\phi$. Let $x \in X$. Then by assumption, $\phi^{-1}(x) \subseteq X^{\prime}$ maps under $\beta$ to a single point of $\Gamma \subseteq X \times_{A} Z$. In particular, (since $\beta$ is surjective) it follows that $\pi: \Gamma \rightarrow X$ is quasi-finite. Since $\pi$ is also projective, it follows that $\pi$ is finite. Since $\pi$ is an isomorphism over $U, \Gamma$ is integral, and $X$ is normal, it thus follows that $\pi$ is an isomorphism. Thus, $f_{U}$ extends to $X$, as desired.

\section{$\S 6$. The Extension Theorem}

If $g$ and $r$ are nonnegative integers such that $2 g-2+r \geq 1$, let us write $\overline{\mathcal{M}}_{g, r} \rightarrow$ $\operatorname{Spec}(\mathbf{Z})$ for the moduli stack of $r$-pointed stable curves of genus $g$ over $\mathbf{Z}$, and $\mathcal{M}_{g, r} \subseteq \overline{\mathcal{M}}_{g, r}$ for the open substack parametrizing smooth curves (cf. [3], [10]). The following is the main result of the present paper:

Theorem 6.1. (Extension Theorem) Let $(X, M)$ be a regular log scheme. Let $U_{X} \subseteq X$ be the interior of $X$, i.e., the open subscheme where the log structure $M$ is trivial. Write $\overline{\mathcal{M}} \stackrel{\text { def }}{=} \overline{\mathcal{M}}_{g, r}, \mathcal{M} \stackrel{\text { def }}{=} \mathcal{M}_{g, r}$. Let $\overline{\mathcal{M}}^{\mathrm{c}}$ be the coarse moduli space associated to $\overline{\mathcal{M}}_{g, r}$. Suppose that we are given a morphism

$$
h_{U_{X}}: U_{X} \rightarrow \overline{\mathcal{M}}
$$

which satisfies $h_{U_{X}}\left(U_{X}\right) \subseteq \mathcal{M}$. Let us denote by $h_{U_{X}}^{\mathrm{c}}: U_{X} \rightarrow \overline{\mathcal{M}}^{\mathrm{c}}$ the composite of $h_{U_{X}}$ with $\overline{\mathcal{M}} \rightarrow \overline{\mathcal{M}}^{\mathrm{c}}$. Then:

(1) If $h_{U_{X}}$ extends over the generic points of $D_{X}$, then: (i) $h_{U_{X}}^{\mathrm{c}}$ extends to $X$; (ii) there exists a surjective, quasi-finite, log étale morphism 
$(Y, N) \rightarrow(X, M)$ (where $(Y, N)$ is log regular) such that $h_{U_{X}}$ extends over $Y$.

(2) If $h_{U_{X}}$ extends over all points of $X$ of height $\leq 2$, and $X$ is a local complete intersection, then $h_{U_{X}}$ extends to $X$.

(3) If for some $l \geq 3$ which is invertible on $X$, $h_{U_{X}}$ admits a level $l$ structure (cf. Definition 5.6), then $h_{U_{X}}$ extends to $X$.

Proof. By Lemma 5.5 (1), it follows that assertion (1) implies assertion (2). Moreover, part (i) of assertion (1) follows immediately from part (ii) of assertion (1); Corollary 5.3; and the technique of étale descent. (Note: Up to étale localization on $X$, one may always assume that the morphism $Y \rightarrow X$ of part (ii) is finite!) Next, let us observe (relative to the proof of part (ii) of assertion (1)) that by the log purity theorem (Theorem 3.3), it follows that after replacing $(X, M)$ by some $(Y, N)$ as in part (ii) of assertion (1), one may always assume that $h_{U_{X}}: U_{X} \rightarrow \mathcal{M}$ admits a level l structure (cf. Definition 5.6) for some prime $l \geq 3$. Thus, in order to complete the proof Theorem 6.1 , it suffices to prove assertion (3).

In order to prove assertion (3), we may assume (by using faithfully flat descent) that $X$ is the spectrum of a complete, local, strictly henselian ring (with closed point $x$ ). Thus, in particular, $X$ is finite-dimensional. The rest of the proof will be by induction on $\operatorname{dim}(X)$. The case $\operatorname{dim}(X)=1$ is trivial. The case $\operatorname{dim}(X)=2$ follows from Corollaries 1.8, 5.3 (of the present paper), together with Theorem 5.1 of [7]. Now let us assume that $\operatorname{dim}(X) \geq 3$. We may thus assume that Theorem 6.1 holds for regular log schemes of dimension $\leq \operatorname{dim}(X)-1$. In particular, we may assume that $h_{U_{X}}$ extends over $X-\{x\}$.

Let $(Y, N) \rightarrow(X, M)$ be a blow-up morphism as in Theorem 2.1. Note that the fact that $h_{U_{X}}$ admits a level $l$ structure (for some prime $l \geq 3$ ) implies that $h_{U_{X}}$ extends over all the generic points of $D_{Y}$ (cf. the discussion following Definition 5.6). Since $D_{Y}$ is a divisor with normal crossings in the regular scheme $Y$, we may thus apply Theorem 5.1 of [7] to conclude that $h_{U_{X}}$ extends over $Y$. Let us denote this extension by $h_{Y}: Y \rightarrow \overline{\mathcal{M}}$.

Let $Z \subseteq Y$ be as in Theorem 2.1. Thus, $U_{Z} \subseteq Z$ is a product of $\mathbf{G}_{m}$ 's over $k \stackrel{\text { def }}{=} k(x)$. Now I claim that (in the language of [7], §3) the topological type of the curve over $U_{Z}$ defined by $\left.h_{Y}\right|_{U_{Z}}$ is locally constant over $U_{Z}$. But this follows by the same argument as that used in [7] (cf. especially $§ 5.2$, the third paragraph of p. 554; §3): Namely, let $u \in U_{Z}$. Then any irreducible component of $D_{Y}$ at $u$ necessarily contains the generic point of $U_{Z}$ (cf. Theorem 2.1). But one knows in general ([7], §3) that the topological type of the curve defined by $h_{Y}$ is locally constant on each of the strata defined by the various intersections of the irreducible components of $D_{Y}$ (in an étale neighborhood of $u$ ). Thus, it follows that the topological type of the curve in question is locally constant over $U_{Z}$. This completes the proof of the claim. 
On the other hand, since $U_{Z}$ is a product of $\mathbf{G}_{m}$ 's over $k$, it follows from Lemma 4.1 of [7] that $\left.h_{Y}^{\mathrm{c}}\right|_{U_{Z}}$ is constant. Thus, $h_{Y}^{\mathrm{c}}$ maps $Z$ to a point. Now since $X$ is normal, the fibers of $Y \rightarrow X$ are connected. Thus, it follows that $h_{Y}^{\mathrm{c}}$ maps $f^{-1}(x)_{\text {red }}$ to a point. Thus, by Lemma 5.7, it follows that $h_{Y}^{\mathrm{c}}$ factors through $X$, i.e., $h_{U_{X}}^{\mathrm{c}}$ extends to a morphism $h_{X}^{\mathrm{c}}: X \rightarrow \overline{\mathcal{M}}^{\mathrm{c}}$. Finally, by Lemma 5.4, we conclude that $h_{Y}$ factors through $X$, i.e., that $h_{U_{X}}$ extends to a morphism $h_{X}: X \rightarrow \overline{\mathcal{M}}$, as desired.

Corollary 6.2. Let $S$ be a noetherian scheme; and $(f: X \rightarrow S, D)$ be polystable (cf. Definition 4.5). Suppose that $\left(X^{\prime}, U^{\prime}\right)$ is a toric pair, and that $X^{\prime}$ has the structure of an S-scheme. Let

$$
h_{U^{\prime}}: U^{\prime} \rightarrow X
$$

be a morphism of $S$-schemes such that $h_{U^{\prime}}\left(U^{\prime}\right) \subseteq U_{X} \stackrel{\text { def }}{=} X-D$. Then $h_{U^{\prime}}$ extends uniquely to a morphism $h_{X^{\prime}}: X^{\prime} \rightarrow X$.

Proof. Suppose that the composite $h_{U^{\prime}}[i+1]: U^{\prime} \rightarrow X_{i+1}$ of $h_{U^{\prime}}$ with $\phi_{i+1}: X \rightarrow X_{i+1}$ extends to a morphism $h_{X^{\prime}}[i+1]: X^{\prime} \rightarrow X_{i+1}$ (for some $i=0, \ldots, n$ ). (For instance, in the case $i=n$, we know that $h_{U^{\prime}}[n+1]$ extends to a morphism $h_{X^{\prime}}[n+1]: X^{\prime} \rightarrow X_{n+1}=S$ - indeed, one can simply take the structure morphism $X^{\prime} \rightarrow S$ for $h_{X^{\prime}}[n+1]$.) Then we would like to show that $h_{U^{\prime}}[i]$ extends to a morphism $h_{X^{\prime}}[i]: X^{\prime} \rightarrow X_{i}$. If we can show the existence of $h_{X^{\prime}}[i]$ whenever $h_{X^{\prime}}[i+1]$ is assumed to exist, then reverse induction on $i$ implies the conclusion of Corollary 6.2. Thus, it suffices to show (for $i=0, \ldots, n$ ) that

(*) If the extension $h_{X^{\prime}}[i+1]: X^{\prime} \rightarrow X_{i+1}$ exists, then the extension $h_{X^{\prime}}[i]: X^{\prime} \rightarrow X_{i}$ exists.

Let us show $\left(^{*}\right)$. First, consider the stable curve $\left(f_{i}: X_{i} \rightarrow X_{i+1}, D_{i}\right)$. This curve defines a classifying morphism $\alpha: X_{i+1} \rightarrow \overline{\mathcal{M}}=\overline{\mathcal{M}}_{g, r}$ (for some $g, r$ ). Moreover, $X_{i} \rightarrow X_{i+1}$ may be identified with the pull-back by $\alpha$ of the tautological curve $\overline{\mathcal{C}} \rightarrow \overline{\mathcal{M}}$, i.e., $X_{i}=\overline{\mathcal{C}} \times \overline{\mathcal{M}}, \alpha_{i+1} X_{i}$. Let $\theta: U^{\prime} \rightarrow \overline{\mathcal{C}}$ be the morphism obtained by composing $U^{\prime} \rightarrow X_{i}$ with the projection $X_{i} \rightarrow \overline{\mathcal{C}}$. Next, observe that $\overline{\mathcal{C}}$ may be identified with $\overline{\mathcal{M}}_{g, r+1}$. Thus, Theorem 6.1 implies that $\theta^{\mathrm{c}}: U^{\prime} \rightarrow \overline{\mathcal{C}}^{\mathrm{c}}$ extends to $X^{\prime}$. But clearly

$$
X_{i}=\overline{\mathcal{C}} \times \overline{\mathcal{M}} X_{i+1} \rightarrow \overline{\mathcal{C}}^{\mathrm{c}} \times \overline{\mathcal{M}}^{\mathrm{c}} X_{i+1}
$$

is a quasi-finite (hence finite) surjective morphism of projective schemes over $X_{i+1}$. Moreover, we have just seen that $U^{\prime} \rightarrow \overline{\mathcal{C}}^{\mathrm{c}} \times \overline{\mathcal{M}}^{\mathrm{c}} X_{i+1}$ extends to $X^{\prime}$. Thus, Corollary 5.3 implies that $U^{\prime} \rightarrow X_{i}$ extends to $X^{\prime}$, as desired. 


\section{$\S 7$. Application to Hyperbolic Polycurves}

Let $\left(S, U_{S}\right)$ be a toric pair. Let

$$
\left(X, U_{X}\right) \rightarrow\left(S, U_{S}\right)
$$

be polystable (cf. Definition 4.5). Then it follows immediately from the definitions (especially property (2) of Definition 4.5) that the morphism between the interiors

$$
h: U_{X} \rightarrow U_{S}
$$

is a hyperbolic polycurve (cf. Definition 4.6).

Definition 7.1. We shall refer to a polyhyperbolic morphism $h: U_{X} \rightarrow U_{S}$ that arises in the above fashion from a polystable $\left(X, U_{X}\right) \rightarrow\left(S, U_{S}\right)$ as compactifiable. Moreover, if $h$ is compactifiable, we shall refer to $\left(X, U_{X}\right) \rightarrow\left(S, U_{S}\right)$ as a compactification of $h$.

Now let $\left(S, U_{S}\right)$ be a toric pair; and let

$$
h: U_{X} \rightarrow U_{S}
$$

be an arbitrary polyhyperbolic morphism. That is to say, we do not assume that $h$ is compactifiable.

In this $\S$, we would like to show that under certain conditions, $h$ necessarily admits a compactification which is "natural" in the sense that it is functorial with respect to certain types of morphisms.

Definition 7.2. Let $U_{X} \rightarrow U_{S}$ be a hyperbolic curve. We shall say that it is split if (cf. Definition 4.4) there exists a stable curve $\left(Y \rightarrow U_{S}, D_{M}\right)$ such that: (i) $Y \rightarrow U_{S}$ is smooth; (ii) $U_{X}$ is $U_{S}$-isomorphic to $Y-D_{M}$.

Let $l$ be a prime number $\geq 3$. We shall say that $U_{X} \rightarrow U_{S}$ is $l$-saturated if it is split, and the corresponding classifying morphism $U_{S} \rightarrow \mathcal{M}_{g, r}$ admits a level $l$ structure (cf. Definition 5.6).

We shall say that $U_{X} \rightarrow U_{S}$ is saturated if there exist (not necessarily distinct) prime numbers $l_{1}, l_{2} \geq 3$ such that: (i) the open subschemes $\left(U_{S}\right)_{1} \stackrel{\text { def }}{=} U_{S}\left[\frac{1}{l_{1}}\right]$ and $\left(U_{S}\right)_{2} \stackrel{\text { def }}{=} U_{S}\left[\frac{1}{l_{2}}\right]$

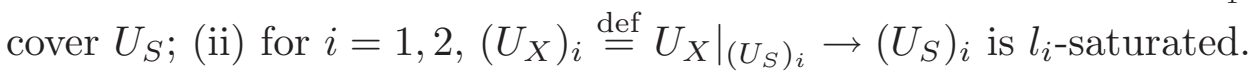


Definition 7.3. Let $U_{X} \rightarrow U_{S}$ be polyhyperbolic. We shall say that it is split (respectively, l-saturated; saturated) if each of the $U_{i} \rightarrow U_{i+1}$ (for $i=0, \ldots, n$ ) of Definition 4.6 is split (respectively, $l$-saturated; saturated).

Corollary 7.4. (Compactification Theorem) Let $\left(S, M_{S}\right)$ be a regular log scheme. Let $U_{S} \subseteq S$ be the interior of $S$. Let $U_{X} \rightarrow U_{S}$ be polyhyperbolic and saturated (cf. Definitions 4.6, 7.3). Then $U_{X} \rightarrow U_{S}$ may be compactified to a (log) polystable $\left(X, M_{X}\right) \rightarrow$ $\left(S, M_{S}\right)$ (cf. Definition 4.5, 7.1).

Moreover, this compactification is natural in the following sense: Suppose that $\left(S^{\prime}, M_{S^{\prime}}\right)$ is a regular log scheme, and let $\left(X, M_{X}\right) \rightarrow\left(S, M_{S}\right)$, and $\left(X^{\prime}, M_{X^{\prime}}\right) \rightarrow\left(S^{\prime}, M_{S^{\prime}}\right)$ be $\log$ polystable with associated hyperbolic polycurves $U_{X} \rightarrow U_{S}$ and $U_{X^{\prime}} \rightarrow U_{S^{\prime}}$, respectively. Suppose, moreover, that we are given a commutative diagram:

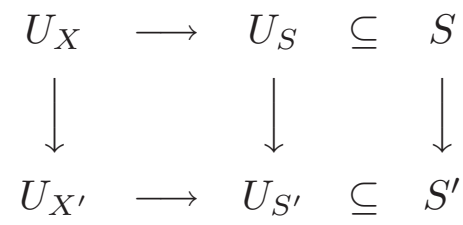

Then the square on the left "compactifies" uniquely to a commutative diagram:

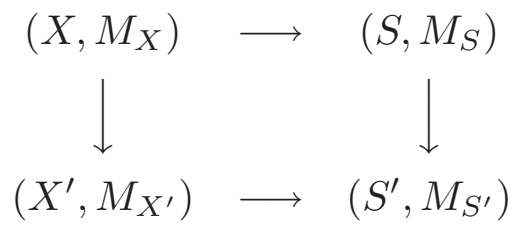

In particular, it makes sense to speak of "the" compactification of $U_{X} \rightarrow U_{S}$.

Proof. First, we prove the existence of a polystable $\left(X, U_{X}\right) \rightarrow\left(S, U_{S}\right)$. We use induction on the " $n$ " appearing in Definition 4.6. Thus, we assume that $U_{1} \rightarrow U_{S}$ has been compactified to some $\left(X_{1}, U_{1}\right) \rightarrow\left(S, U_{S}\right)$. Moreover, we have a (split) saturated hyperbolic curve $U_{0} \rightarrow U_{1}$. This curve gives us a classifying morphism

$$
U_{1} \rightarrow \mathcal{M} \subseteq \overline{\mathcal{M}} \stackrel{\text { def }}{=} \overline{\mathcal{M}}_{g, r}
$$

(for some $g, r$ ). Because of the naturality assertion in Theorem 7.4, it suffices to construct $(X \rightarrow S, D)$ locally over $\operatorname{Spec}(\mathbf{Z})$. Thus, the saturatedness assumption implies that we may assume that $U_{1} \rightarrow \overline{\mathcal{M}}$ admits a level $l$ structure (for some prime $l \geq 3$ ) - cf. Definition 5.6. Since $\left(X_{1}, U_{1}\right)$ is toric (by Lemma 4.2), applying Theorem 6.1 implies that this morphism extends to a morphism $X_{1} \rightarrow \overline{\mathcal{M}}$. Pulling back the universal curve and divisor of marked points over $\overline{\mathcal{M}}$, we thus obtain a compactification $\left(X, U_{X}\right) \rightarrow\left(S, U_{S}\right)$, as desired. 
Now we consider the naturality assertion. The point here is that $\left(X^{\prime}, U_{X^{\prime}}\right) \rightarrow\left(S^{\prime}, U_{S^{\prime}}\right)$ is polystable, while $\left(X, U_{X}\right)$ is toric. Moreover, $X$ has an $S^{\prime}$-scheme structure such that all the morphisms involved are $S^{\prime}$-morphisms. Thus, we may apply Corollary 6.2 to conclude that we get a morphism from $X \rightarrow S$ to $X^{\prime} \rightarrow S^{\prime}$, as desired. This completes the proof of Theorem 7.4.

We conclude this paper with some remarks concerning Corollary 7.4.

Remark 1. Concerning coverings of hyperbolic polycurves: In characteristic 0, it is easy to see that any connected, finite, étale cover of a hyperbolic polycurve is again a hyperbolic polycurve. Thus, given an arbitrary polyhyperbolic $U \rightarrow T$, there exists a polyhyperbolic $U^{\prime} \rightarrow T^{\prime}$ such that $U^{\prime}$ (respectively, $T^{\prime}$ ) is finite étale over $U$ (respectively, $T$ ) and such that $U^{\prime} \rightarrow T^{\prime}$ is (split and) saturated. In characteristic $p$ or mixed characteristic, however, finite étale covers of hyperbolic polycurves need not be hyperbolic polycurves. Thus, when the base is not of characteristic 0 , it is often a nontrivial task to construct hyperbolic polycurves satisfying the hypotheses of Theorem 5.4.

Remark 2. Concerning the "Saturatedness" Hypothesis in Corollary 7.4: This hypothesis may strike the reader as being unnecessarily strong since there certainly exist many compactifiable hyperbolic polycurves which are not saturated. On the other hand, from another point of view, this hypothesis is, in fact, rather natural, and not so restrictive at all: Indeed, if a hyperbolic polycurve $U_{X} \rightarrow U_{S}$ admits a compactification $\left(X, U_{X}\right) \rightarrow\left(S, U_{S}\right)$, then the $l$-torsion points (for $l$ invertible on $S$ ) of each hyperbolic curve in a sequence as in Definition 4.6 are tamely ramified over the base (i.e., the next higher numbered curve in a sequence as in Definition 4.6). Thus, the compactification $\left(X, U_{X}\right) \rightarrow\left(S, U_{S}\right)$ necessarily fits into a commutative (in general, non-cartesian) diagram:

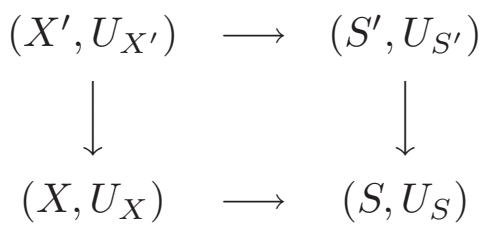

where the vertical arrows are finite, Galois, and log étale, and $U_{X^{\prime}} \rightarrow U_{S^{\prime}}$ is a saturated hyperbolic polycurve (i.e., is of the sort that is treated in the existence part of Corollary 7.4). In fact, if the Galois groups of the vertical arrows are $G_{X}, G_{S}$, respectively, then (by the functoriality part of Corollary 7.4) $G_{X}$ and $G_{S}$ act on the above commutative diagram. Thus, if we form the quotient of the above commutative diagram by these actions in the sense of stacks, we obtain a commutative diagram

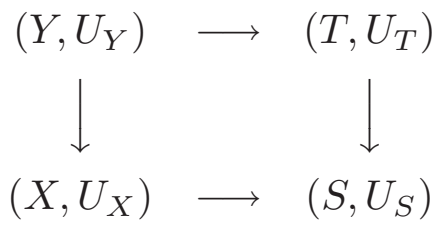


whose vertical arrows are now proper, quasi-finite, log étale, and birational (even isomorphisms over $U_{X}$ and $\left.U_{S} !\right)$. (Note that since, in general, $Y$ and $T$ are algebraic stacks, "proper + quasi-finite $\neq$ finite.") Thus, in summary, although there exist compactifiable hyperbolic polycurves not covered by the existence part of Corollary 7.4, modulo the operation of putting an appropriate stack structure on the compactification $X \rightarrow S$ at the boundaries $X-U_{X}, S-U_{S}$, every compactifiable hyperbolic polycurve is, in fact, covered by Corollary 7.4.

Remark 3. Relationship to de Jong's theory of alterations: Corollary 7.4 gives another way to think about de Jong's work ([6]) on alterations. For instance, suppose that $R$ is a Dedekind domain whose quotient field $K$ we assume (for simplicity) to be of characteristic 0 . Let $X_{K}$ be a variety over $K$ (i.e., a geometrically irreducible $K$-scheme). It is a wellknown fact in algebraic geometry that any smooth point of $X_{K}$ admits a neighborhood $U_{K} \subseteq X_{K}$ such that $U_{K}$ is an "Artin neighborhood." In our terminology, by taking $U_{K}$ to be sufficiently small, one may assume that $U_{K}$ is a hyperbolic polycurve over $K$. In fact, if we do not specify a point of $X_{K}$ that we wish to have belong to $U_{K}$, then it is an easy exercise in elementary commutative algebra to show that such a $U_{K}$ exists. Then applying Corollary 7.4 (cf. also Remarks 1 and 2 above), we see immediately that there exists a proper algebraic stack (which one can think of as a "stack-alteration" of $X_{K}$ )

$$
\mathcal{Z} \rightarrow \operatorname{Spec}(R)
$$

such that: (i) (when equipped with an appropriate log structure) $\mathcal{Z}$ is $\log$ regular; (ii) $U_{K}$ is an open subscheme of $\mathcal{Z}_{K} \stackrel{\text { def }}{=} \mathcal{Z} \otimes_{R} K$.

In fact, given an arbitrary proper model $\mathcal{X} \rightarrow \operatorname{Spec}(R)$ of $X_{K}$ over $R$, by taking $U_{K}$ to be sufficiently small, one can even assume that $\mathcal{Z}$ is equipped with a proper $R$-morphism

$$
\mathcal{Z} \rightarrow \mathcal{X}
$$

(which is the identity on $U_{K}$ ). Indeed, this is precisely the sort of "resolution of indeterminacy" problem that is already solved by Corollary 7.4: First of all, we may cover $\mathcal{X}$ by affines $\left\{\mathcal{U}_{\alpha}\right\}$, and assume that each $\mathcal{U}_{\alpha}$ is embedded in some affine space over $R$. But affine space (which is just a product of $\mathbf{A}^{1}$ 's) may be regarded as a partial compactification of a product of $\mathbf{P}^{1}-\{0,1, \infty\}$ 's, which are hyperbolic curves! Thus, by taking $U_{K} \subseteq X_{K}$ to be sufficiently small - i.e., so small that it is contained in all the $\mathcal{U}_{\alpha}$ 's, and, moreover, (relative to the affine embedding of each $\mathcal{U}_{\alpha}$ ) maps into the open subscheme of affine space given by a product of $\mathbf{P}^{1}-\{0,1, \infty\}$ 's - we obtain that the functoriality portion of Corollary 7.4 implies that we get a proper $R$-morphism $\mathcal{Z} \rightarrow \mathcal{X}$, as desired.

In fact, by arguing as above, Corollary 7.4 can be used to give proofs of many of the results of [6] (cf., e.g., [15] for more details).

Thus, in summary, although Corollary 7.4 appears to address only certain specific compactification/resolution of indeterminacy problems (i.e., those concerning hyperbolic 
polycurves), in fact, because of the ubiquity of hyperbolic polycurves in algebraic geometry - e.g., in the form of Artin neighborhoods; as the open subscheme $\left(\mathbf{P}^{1}-\{0,1, \infty\}\right)^{N} \subseteq$ $\left(\mathbf{A}^{1}\right)^{N}=\mathbf{A}^{N}$ in affine space - Corollary 7.4 can be applied to a fairly large and diverse collection of compactification/resolution of indeterminacy problems in algebraic geometry.

\section{Bibliography}

[1] D. Abramovich and A. Vistoli, Complete Moduli for Fibered Surfaces, preprint (December 1997).

[2] M. Deschamps, Réduction semi-stable in L. Szpiro, Séminaire sur les pinceaux de courbes de genre au moins deux, Astérisque 86 (1981).

[3] P. Deligne and D. Mumford, The Irreducibility of the Moduli Space of Curves of Given Genus, IHES Publ. Math. 36 (1969), 75-109.

[4] G. Faltings and C.-L. Chai, Degenerations of Abelian Varieties, Springer (1990).

[5] K. Fujiwara and K. Kato, Log Etale Topology Theory, preprint.

[6] A. J. de Jong, Smoothness, Semi-stability, and Alterations, IHES Publ. Math 83 (1996), 51-93.

[7] A. J. de Jong and F. Oort, On Extending Families of Curves, Journal of Alg. Geom. 6 (1997), 545-562.

[8] K. Kato, Logarithmic Structures of Fontaine-Illusie, Proceedings of the First JAMI Conference, Johns Hopkins Univ. Press (1990), 191-224.

[9] K. Kato, Toric Singularities, Amer. J. Math. 116 (1994), 1073-1099.

[10] F. F. Knudsen, The Projectivity of the Moduli Space of Stable Curves, II, Math. Scand. 52 (1983), 161-199.

[11] R. Hartshorne, Algebraic Geometry, Graduate Texts in Math. 52 (1997), Springer.

[12] R. Hartshorne, Residues and Duality, Lect. Notes in Math. 20 (1966), Springer.

[13] H. Matsumura, Commutative Algebra (Second Edition), The Benjamin/Cummings Publishing Company (1980).

[14] S. Mochizuki, The Geometry of the Compactification of the Hurwitz Scheme, Publ. RIMS, Kyoto Univ. 31, No. 3 (1995), 355-441.

[15] S. Mochizuki, Extending Families of Curves I: Compactification of Hyperbolic Polycurves, RIMS Preprint 1188 (March 1998).

[16] D. Mumford, Stability of Projective Varieties, Enseignement Math. (2) 23, No. 1-2 (1977), 39-110.

[17] T. Oda, Convex Bodies and Algebraic Geometry: An Introduction to the Theory of Toric Varieties, Springer (1988). 
[18] A. Grothendieck et al., Cohomologie locale des faisceaux cohérents et théorèmes de Lefshetz locaux et globaux, North-Holland, Amsterdam (1968).

Shinichi Mochizuki

Research Institute for Mathematical Sciences

Kyoto University

Kyoto 606-01, JAPAN

motizuki@kurims.kyoto-u.ac.jp 